ESAIM: COCV 27 (2021) 92

https://doi.org/10.1051/cocv/2021086
ESAIM: Control, Optimisation and Calculus of Variations

www.esaim-cocv.org

\title{
MULTI-TIME STATE MEAN-VARIANCE MODEL IN CONTINUOUS TIME*
}

\author{
SHUZHEN YANG**
}

\begin{abstract}
The objective of the continuous time mean-variance model is to minimize the variance (risk) of an investment portfolio with a given mean at the terminal time. However, the investor can stop the investment plan at any time before the terminal time. To solve this problem, we consider to minimize the variances of the investment portfolio in the multi-time state. The advantage of this multi-time state mean-variance model is the minimization of the risk of the investment portfolio within the investment period. To obtain the optimal strategy of the model, we introduce a sequence of Riccati equations, which are connected by jump boundary conditions. In addition, we establish the relationships between the means and variances in the multi-time state mean-variance model. Furthermore, we use an example to verify that the variances of the multi-time state can affect the average of Maximum-Drawdown of the investment portfolio.
\end{abstract}

Mathematics Subject Classification. 91B28, 93E20, 49N10.

Received June 5, 2020. Accepted August 28, 2021.

\section{INTRODUCTION}

In the financial market, standard deviation or variance is always used to measure the risk of a given risky asset. Variance is an important metric in the investment, which can help an investor determine whether it will be profitable. Investors can achieve the best asset allocation in a portfolio by analyzing the variance of the returns among the assets in the portfolio. Semi-deviation is proposed to capture the downside risk of an investment which focuses only on negative price fluctuations. Conversely, variance treats all deviations from the mean as the same regardless of their direction. Thus, in this paper, we use variance to measure the risk of an investment.

To balance the return (mean) and risk (variance) in a single-period portfolio selection model, references [19, 20] proposed the mean-variance model. Since then, many related works focused on these topics. Under some mild assumptions, [22] solved the single-period problem analytically. Reference [24] studied a mean-variance model in which a single stock with a constant risk-free rate was introduced. Dynamic asset allocation in a meanvariance framework was studied by [1]. Reference [16] embedded the discrete-time multi-period mean-variance problem within a multi-objective optimization framework and obtained an optimal strategy. By extending the embedding technique introduced in [16] and applying the results from the stochastic linear quadratic control

* This work was supported by the National Key R\&D Program of China (No. 2018YFA0703900) and National Natural Science Foundation of China (No. 11701330; 11871050) and Young Scholars Program of Shandong University.

Keywords and phrases: Mean-variance, multi-time state, stochastic optimal control.

Shandong University-Zhong Tai Securities Institute for Financial Studies, Shandong University, Jinan, PR China.

** Corresponding author: yangsz@sdu.edu.cn 
in the continuous time case, [33] investigated an optimal pair for the continuous-time mean-variance problem. Further results in the mean-variance problem included those with bankruptcy prohibition, transaction costs, and random parameters in complete and incomplete markets, see $[5,9,17,18,27]$. The aforementioned precommitted strategies in the continuous time multi-period, differed from those of the single-period case. For further details, see [15].

The optimal strategy in the multi-period mean-variance framework is a pre-committed strategy that strengths the premise that the investor needs to follow the strategy given at the initial time. When the optimal strategy is not time-consistent, the investor may not obey this strategy in the following investment period. A game theoretic approach to study the time-inconsistency of the mean-variance model was developed in [2]. Reference [11] considered a general time-inconsistent stochastic linear-quadratic control problem with an equilibrium instead of optimal control. Based on a local mean-variance efficiency, [8] developed a time-consistent formulation of the mean-variance portfolio selection problem in a general semi-martingale setting. Subsequently, the meanvariance portfolio selection problem in a complete market with unbounded random coefficients was studied in [25]. The large population stochastic dynamic games and the Nash certainty equivalence based on control laws were established in [12]. Based on the adjoint equation approach, [4] studied the linear-quadratic mean field games (see [3]). The mean-variance problem with state dependent risk aversion was considered in [6]. A general framework to study the time-inconsistent stochastic control in the continuous time framework was established in [7]. In addition, [23] investigated a robust time-inconsistent stochastic control problem, and applied it to the robust dynamic mean-variance portfolio selection problem. In the incomplete markets with stochastic volatility, [28] considered the open-loop control framework for time-consistent mean-variance portfolio problems. A dynamic mean-variance analysis for log returns within the game-theoretic approach was proposed in [10]. Further, [13] introduced a "dynamic utility" under which the original time-inconsistent problem became a time-consistent one, and investigated possible approaches to study a general time-inconsistent optimization problem. Reference [14] used a time-consistent dynamic convex risk measure to evaluate the risk of a portfolio and showed that the dynamic mean-risk problem satisfies a set-valued Bellman's principle.

In the financial market, for a given terminal time $T, Y^{\pi}(T)$ represents a portfolio asset with strategy $\pi(\cdot)$, while $\mathbb{E}\left[Y^{\pi}(T)\right]$ and $\operatorname{Var}\left(Y^{\pi}(T)\right)=\mathbb{E}\left(Y^{\pi}(T)-\mathbb{E}\left[Y^{\pi}(T)\right]\right)^{2}$ represent the mean and variance of $Y^{\pi}(T)$, respectively. In the classical mean-variance model, we want to minimize the variance of the portfolio asset $\operatorname{Var}\left(Y^{\pi}(T)\right)$ for a given mean $\mathbb{E}\left[Y^{\pi}(T)\right]=L$, where $L$ is a constant. The investor can stop the investment plan at an uncertain horizon time $\tau$ before the terminal time $T$, where $\tau \leq T$. Therefore, there are many related works on the meanvariance portfolio model with an uncertain horizon time. Reference [21] considered static mean-variance analysis with an uncertain time horizon. Moreover, [31] studied the mean-variance model of a multi-period asset-liability management problem under uncertain exit time; see [26, 30, 32] for additional studies in this vein. However, in the literature of mean-variance model under uncertain or random exit time, authors always suppose that the uncertain horizon time $\tau$ satisfies a distribution (or a conditional distribution) and investigate the related mean-variance model at time $\tau$.

However, in general, we do not know the information of $\tau$ at initial time $t_{0}=0$. Given a probability space $(\Omega, \mathcal{F}, P)$, notice that for a given partition $0=t_{0}<t_{1}<\cdots<t_{N}=T$ of interval $[0, T]$ and $\omega \in \Omega$, there exists $i \in\{0,1, \ldots, N-1\}$ such that $\tau(\omega) \in\left[t_{i}, t_{i+1}\right]$. To reduce the variance of the portfolio asset $Y^{\pi}(\cdot)$ at $\tau \in(0, T]$, we consider minimizing the variances of the portfolio asset at multi-time state $\left(Y^{\pi}\left(t_{1}\right), Y^{\pi}\left(t_{2}\right), \ldots, Y^{\pi}\left(t_{N}\right)\right)$ with constraint on means of multi-time state $\left(Y^{\pi}\left(t_{1}\right), Y^{\pi}\left(t_{2}\right), \ldots, Y^{\pi}\left(t_{N}\right)\right)$. Therefore, we introduce the following multi-time state mean-variance model:

$$
J(\pi(\cdot))=\sum_{i=1}^{N} \alpha_{i} \mathbb{E}\left(Y^{\pi}\left(t_{i}\right)-\mathbb{E}\left[Y^{\pi}\left(t_{i}\right)\right]\right)^{2},
$$

where $\alpha_{i} \in[0,1]$ and $\sum_{i=1}^{N} \alpha_{i}=1$, with constraints on the multi-time state means,

$$
\mathbb{E}\left[Y^{\pi}\left(t_{i}\right)\right]=L_{i}, i=1,2, \ldots, N
$$


In this multi-time state mean-variance model, we can minimize the risk of the investment portfolio within the multi-time $\left(t_{1}, t_{2}, \ldots, t_{N}\right)$. Note that the multi-time state $\left(Y^{\pi}\left(t_{1}\right), Y^{\pi}\left(t_{2}\right), \ldots, Y^{\pi}\left(t_{N}\right)\right)$ of the investment portfolio can affect the value of each other, and we cannot solve the multi-time state mean-variance model via one classical Riccati equation directly. To obtain the optimal strategy of the multi-time state mean-variance model, we introduce a sequence of Riccati equations, which are connected by jump boundary conditions (see Eqs. (3.5) and (3.6)). Based on this sequence of Riccati equations, we investigate an optimal strategy (see Thm. 3.2) and establish the relationships between the means and variances of this multi-time state mean-variance model (see Lem. 3.4).

The Maximum-Drawdown of the asset $Y^{\pi}(\cdot)$ is an important index to evaluate a strategy in the investment portfolio model, where the Maximum-Drawdown of the asset $Y^{\pi}(\cdot)$ is defined in the interval $[0, h], h \leq T$, by

$$
\operatorname{MD}_{Y^{\pi}}^{h}=\operatorname{esssup}\left\{z \mid z=Y^{\pi}(t)-Y^{\pi}(s), 0 \leq t \leq s \leq h\right\}
$$

Based on the simulation results of the multi-time state mean-variance model (see subsection 4.2), we can see that the constrained condition (1.2) can affect the average of $\mathrm{MD}_{Y^{\pi}}^{h}$ of the portfolio asset $Y^{\pi}(\cdot)$ (see Fig. 3). This study is most closely related to the study of [29], in which the author established the necessary and sufficient conditions for stochastic differential systems with multi-time state cost functional.

The remainder of this paper is organized as follows. In Section 2, we formulate the multi-time state meanvariance model. Subsequently, in Section 3, we investigate an optimal strategy and establish the relationships between multi-time state means and variances for the proposed model. In Section 4, based on the main results of Section 3, we compare the multi-time state mean-variance model with the classical mean-variance model. Finally, we conclude the paper in Section 5.

\section{Multi-Time State MEAN-VARIANCE MODEL}

Let $W(\cdot)$ be a $d$-dimensional standard Brownian motion defined on a complete filtered probability space $\left(\Omega, \mathcal{F}, P ;\{\mathcal{F}(t)\}_{t \geq 0}\right)$, where $\{\mathcal{F}(t)\}_{t \geq 0}$ is the $P$-augmentation of the natural filtration generated by $W(\cdot)$. There is one risk-free bond asset and $n$ risky stock assets that are traded in the market, where the bond satisfies the following equation:

$$
\left\{\begin{aligned}
\mathrm{d} R_{0}(t) & =r(t) R_{0}(t) \mathrm{d} t, \quad t>0, \\
R_{0}(0) & =a_{0}>0
\end{aligned}\right.
$$

and the $i$-th $(1 \leq i \leq n)$ stock asset is described by

$$
\left\{\begin{array}{l}
\mathrm{d} R_{i}(t)=b_{i}(t) R_{i}(t) \mathrm{d} t+R_{i}(t) \sum_{j=1}^{d} \sigma_{i j}(t) \mathrm{d} W_{j}(t), \quad t>0, \\
R_{i}(0)=a_{i}>0,
\end{array}\right.
$$

where $r(\cdot) \in \mathbb{R}$ is the risk-free return rate of the bond, $b(\cdot)=\left(b_{1}(\cdot), \ldots, b_{n}(\cdot)\right) \in \mathbb{R}^{n}$ is the expected return rate of the risky assets, and $\sigma(\cdot)=\left(\sigma_{1}(\cdot), \ldots, \sigma_{n}(\cdot)\right)^{\top} \in \mathbb{R}^{n \times d}$ is the corresponding volatility matrix. Given a initial capital $y>0, \gamma(\cdot)=\left(\gamma_{1}(\cdot), \ldots, \gamma_{n}(\cdot)\right) \in \mathbb{R}^{n}$, where $\gamma_{i}(\cdot)=b_{i}(\cdot)-r(\cdot), 1 \leq i \leq n$. The investor's wealth $Y^{\pi}(\cdot)$ satisfies

$$
\left\{\begin{aligned}
\mathrm{d} Y^{\pi}(t) & =\left[r(t) Y^{\pi}(t)+\gamma(t) \pi(t)^{\top}\right] \mathrm{d} t+\pi(t) \sigma(t) \mathrm{d} W(t) \\
Y^{\pi}(0) & =y
\end{aligned}\right.
$$


where $\pi(\cdot)=\left(\pi_{1}(\cdot), \ldots, \pi_{n}(\cdot)\right) \in \mathbb{R}^{n}$ is the capital invested in the risky assets $R(\cdot)=\left(R_{1}(\cdot), \ldots, R_{n}(\cdot)\right) \in \mathbb{R}^{n}$ and $\pi_{0}(\cdot)$ is that invested in the bond. Thus, we have

$$
Y^{\pi}(\cdot)=\sum_{i=0}^{n} \pi_{i}(\cdot)
$$

In this study, we consider the following multi-time state mean-variance model:

$$
J_{1}(\pi(\cdot))=\sum_{i=1}^{N} \alpha_{i} \mathbb{E}\left[Y^{\pi}\left(t_{i}\right)-\mathbb{E}\left[Y^{\pi}\left(t_{i}\right)\right]\right]^{2},
$$

with constraint on the multi-time state mean,

$$
\mathbb{E}\left[Y^{\pi}\left(t_{i}\right)\right]=L_{i}, i=1,2, \ldots, N,
$$

where $0=t_{0}<t_{1}<\cdots<t_{N}=T, \alpha_{i} \in[0,1]$ and $\sum_{i=1}^{N} \alpha_{i}=1$.

Remark 2.1. In the multi-time state mean-variance model $(2.2), J_{1}(\pi(\cdot))$ generalizes the traditional meanvariance model:

(i). When $\alpha_{i}=0,1 \leq i \leq N-1$ and $\alpha_{N}=1, J_{1}(\pi(\cdot))$ becomes the classical mean-variance model;

(ii). For a given stopping time $\tau \in(0, T]$ independent from Brownian motion $W(\cdot)$, we can take $\alpha_{i}=P(\tau \in$ $\left.\left(t_{i-1}, t_{i}\right]\right), 1 \leq i \leq N$. Subsequently, $J_{1}(\pi(\cdot))$ can be used to approximate the variance at $\tau$, $\operatorname{Var}\left(Y^{\pi}(\tau)\right)=$ $\mathbb{E}\left(Y^{\pi}(\tau)-\mathbb{E}\left[Y^{\pi}(\tau)\right]\right)^{2}$

(iii). Let $N=2$. In the classical mean-variance model, we can rewrite the variance at terminal time $t_{2}$ as $\operatorname{Var}\left(Y^{\pi}\left(t_{2}\right)\right)=\operatorname{Var}\left(Y^{\pi}\left(t_{2}\right)-Y^{\pi}\left(t_{0}\right)\right)$. Thus, we can consider the variance between $t_{1}$ and $t_{2}$, that is $\operatorname{Var}\left(Y^{\pi}\left(t_{2}\right)-Y^{\pi}\left(t_{1}\right)\right)$. Note that, when the covariance of $Y^{\pi}\left(t_{2}\right)$ and $Y^{\pi}\left(t_{1}\right)$ is 0 , we have $\operatorname{Var}\left(Y^{\pi}\left(t_{2}\right)-Y^{\pi}\left(t_{1}\right)\right)=$ $\operatorname{Var}\left(Y^{\pi}\left(t_{2}\right)\right)+\operatorname{Var}\left(Y^{\pi}\left(t_{1}\right)\right)$, which is a special case of the multi-time state mean-variance model (2.2).

The set of admissible strategies $\pi(\cdot)$ is defined as:

$$
\mathcal{A}=\left\{\pi(\cdot): \pi(\cdot) \in L_{\mathcal{F}}^{2}\left[0, T ; \mathbb{R}^{n}\right]\right\}
$$

where $L_{\mathcal{F}}^{2}\left[0, T ; \mathbb{R}^{n}\right]$ is the set of all square integrable measurable $\mathbb{R}^{n}$ valued $\left\{\mathcal{F}_{t}\right\}_{t \geq 0}$ adaptive processes. If there exists a strategy $\pi^{*}(\cdot) \in \mathcal{A}$ that yields the minimum value of the cost functional (2.2), then we say that the multi-time state mean-variance model (2.2) is solved.

We make the following assumptions to obtain the optimal strategy for the proposed model (2.2):

$\mathbf{H}_{1}: r(\cdot), b(\cdot)$ and $\sigma(\cdot)$ are the bounded deterministic continuous functions.

$\mathbf{H}_{2}: r(\cdot), \gamma(\cdot)>0, \sigma(\cdot) \sigma(\cdot)^{\top}>\delta \mathbf{I}$, where $\delta>0$ is a given constant, and $\mathbf{I}$ is the identity matrix of $\mathbb{S}^{n} ; \mathbb{S}^{n}$ is the set of symmetric matrices.

\section{Optimal StRategy}

In this section, we investigate an optimal strategy $\pi(\cdot)$ for the problem defined in $(2.2)$, with a constraint on the multi-time state mean (2.3). Here, we describe how to construct an optimal strategy for (2.2) with constrained condition $(2.3)$. 
Similar to [33], we introduce the following multi-time state mean-variance problem: minimizing the cost functional,

$$
J_{2}(\pi(\cdot))=\sum_{i=1}^{N}\left(\frac{\mu_{i}}{2} \operatorname{Var}\left(Y^{\pi}\left(t_{i}\right)\right)-\mathbb{E}\left[Y^{\pi}\left(t_{i}\right)\right]\right),
$$

where $\mu_{i}=\kappa_{i} \alpha_{i}, 1 \leq i \leq N$, and $\kappa_{i} \neq 0$ is used to combine the mean and variance at time $t_{i}$. To solve the cost functional (3.1), we employ the following model:

$$
J_{3}(\pi(\cdot))=\sum_{i=1}^{N} \mathbb{E}\left[\frac{\mu_{i}}{2} Y^{\pi}\left(t_{i}\right)^{2}-\lambda_{i} Y^{\pi}\left(t_{i}\right)\right] .
$$

Note that, when $\alpha_{i}=0$ at some $1 \leq i \leq N$, we need to delete the $i$-th term. For notation simplicity, we consider $\mu_{i} \neq 0$ and $\lambda_{i} \in \mathbb{R}, i=1,2, \ldots, N$.

For the given $\mu_{i}, i=1,2, \ldots, N$, we suppose $\pi^{*}(\cdot)$ is an optimal strategy of cost functional (3.1). Based on Theorem 3.1 of [33], taking $\lambda_{i}=1+\mu_{i} \mathbb{E}\left[Y^{\pi^{*}}\left(t_{i}\right)\right], i=1,2, \ldots, N$, we can show that $\pi^{*}(\cdot)$ is an optimal strategy of cost functional (3.2). Conversely, let $\pi^{*}(\cdot)$ be an optimal strategy of cost functional (3.2). When $\lambda_{i}=1+\mu_{i} \mathbb{E}\left[Y^{\pi^{*}}\left(t_{i}\right)\right], i=1,2, \ldots, N$, we can show that $\pi^{*}(\cdot)$ is an optimal strategy of cost functional (3.1).

Remark 3.1. In the cost functional (3.1), the weights $\left\{\alpha_{i}\right\}_{i=1}^{N}$ are absorbed within $\left\{\mu_{i}\right\}_{i=1}^{N}$. Then, we embed cost functional (3.1) into (3.2). Furthermore, (3.1) and (3.2) admit the same optimal strategy $\pi^{*}(\cdot)$, when $\lambda_{i}=1+\mu_{i} \mathbb{E}\left[Y^{\pi^{*}}\left(t_{i}\right)\right], i=1,2, \ldots, N$. Thus, the weights $\left\{\alpha_{i}\right\}_{i=1}^{N}$ are included within $\left\{\lambda_{i}\right\}_{i=1}^{N}$ via $\left\{\mu_{i}\right\}_{i=1}^{N}$.

It should be noted that, we cannot solve the cost functional (3.2) by applying the embedding technique of [33] for the multi-time state mean-variance model via the classical Riccati equation directly, as the value $Y^{\pi}\left(t_{i}\right)$ can affect $Y^{\pi}\left(t_{i+1}\right)$, for $i=0,1, \ldots, N-1$.

Denoting

$$
\begin{aligned}
& \rho_{i}=\frac{\lambda_{i}}{\mu_{i}}, z_{i}^{\pi}(t)=Y^{\pi}(t)-\rho_{i}, t_{i-1} \leq t \leq t_{i}, i=1,2, \ldots, N \\
& \beta(t)=\gamma(t)\left[\sigma(t) \sigma(t)^{\top}\right]^{-1} \gamma(t)^{\top}, t \leq T .
\end{aligned}
$$

Thus, the cost functional (3.2) is equivalent to

$$
J_{4}(\pi(\cdot))=\sum_{i=1}^{N} \mathbb{E}\left[\frac{\mu_{i}}{2} z_{i}^{\pi}\left(t_{i}\right)^{2}\right]
$$

where $z_{i}^{\pi}(\cdot)$ satisfies

$$
\left\{\begin{aligned}
\mathrm{d} z_{i}^{\pi}(t) & =\left[r(t) z_{i}^{\pi}(t)+\gamma(t) \pi(t)^{\top}+\rho_{i} r(t)\right] \mathrm{d} t+\pi(t) \sigma(t) \mathrm{d} W(t) \\
z_{i}^{\pi}\left(t_{i-1}\right) & =Y^{\pi}\left(t_{i-1}\right)-\rho_{i}, t_{i-1}<t \leq t_{i}
\end{aligned}\right.
$$

Now, we construct a sequence of Riccati equations that are connected by jump boundary conditions, in which the jump boundary condition can offset the interaction of $Y^{\pi}\left(t_{i+1}\right)$ and $Y^{\pi}\left(t_{i}\right)$, for $i=0,1, \ldots, N-1$. We first introduce a sequence of deterministic Riccati equations:

$$
\left\{\begin{aligned}
\mathrm{d} P_{i}(t) & =[\beta(t)-2 r(t)] P_{i}(t) \mathrm{d} t \\
P_{i}\left(t_{i}\right) & =\mu_{i}+P_{i+1}\left(t_{i}\right), t_{i-1} \leq t<t_{i}, i=1,2, \ldots, N
\end{aligned}\right.
$$


and related equations,

$$
\left\{\begin{aligned}
\mathrm{d} g_{i}(t) & =\left[(\beta(t)-r(t)) g_{i}(t)-\rho_{i} r(t) P_{i}(t)\right] \mathrm{d} t, \\
g_{i}\left(t_{i}\right) & =g_{i+1}\left(t_{i}\right)+P_{i+1}\left(t_{i}\right)\left(\rho_{i}-\rho_{i+1}\right), t_{i-1} \leq t<t_{i}, i=1,2, \ldots, N,
\end{aligned}\right.
$$

where $P_{N+1}\left(t_{N}\right)=0, g_{N+1}\left(t_{N}\right)=0, \rho_{N+1}=0$. Furthermore, by a simple calculation, we have

$$
\frac{g_{i}(t)}{P_{i}(t)}=\frac{g_{i}\left(t_{i}\right)}{P_{i}\left(t_{i}\right)} e^{-\int_{t}^{t_{i}} r(s) \mathrm{d} s}+\rho_{i}\left(1-e^{-\int_{t}^{t_{i}} r(s) \mathrm{d} s}\right), t_{i-1} \leq t \leq t_{i} i=1,2, \ldots, N,
$$

which is used to obtain the following results.

Theorem 3.2. Let Assumptions $\boldsymbol{H}_{1}$ and $\boldsymbol{H}_{2}$ hold, there exists an optimal strategy $\pi^{*}(\cdot)$ for cost functional (3.3), where the optimal strategy $\pi^{*}(\cdot)$ is given as follows:

$$
\pi^{*}(t)=\gamma(t)\left(\sigma(t) \sigma(t)^{\top}\right)^{-1}\left[\left(\rho_{i}-\frac{g_{i}\left(t_{i}\right)}{P_{i}\left(t_{i}\right)}\right) e^{-\int_{t}^{t_{i}} r(s) \mathrm{d} s}-Y^{*}(t)\right], t_{i-1}<t \leq t_{i}
$$

where $Y^{*}(t)=z^{\pi^{*}}(t)+\rho_{i}, t_{i-1}<t \leq t_{i}$ and $i=1,2, \ldots, N$.

Proof. For any given $i \in\{1,2, \cdots, N\}, t_{i-1}<t \leq t_{i}$, applying Itô formula to $z_{i}^{\pi}(t)^{2} P_{i}(t)$, we have

$$
\begin{aligned}
& \frac{1}{2} \mathrm{~d} z_{i}^{\pi}(t)^{2} P_{i}(t) \\
= & \frac{1}{2}\left\{2 z_{i}^{\pi}(t) P_{i}(t)\left[r(t) z_{i}^{\pi}(t)+\gamma(t) \pi(t)^{\top}+\rho_{i} r(t)\right]+z_{i}^{\pi}(t)^{2}[\beta(t)-2 r(t)] P_{i}(t)\right. \\
& \left.+P_{i}(t) \pi(t) \sigma(t) \sigma(t)^{\top} \pi(t)^{\top}\right\} \mathrm{d} t+z_{i}^{\pi}(t) P_{i}(t) \pi(t) \sigma(t) \mathrm{d} W(t) \\
= & \frac{1}{2}\left\{2 z_{i}^{\pi}(t) P_{i}(t)\left[\gamma(t) \pi(t)^{\top}+\rho_{i} r(t)\right]+z_{i}^{\pi}(t)^{2} \beta(t) P_{i}(t)\right. \\
& \left.+P_{i}(t) \pi(t) \sigma(t) \sigma(t)^{\top} \pi(t)^{\top}\right\} \mathrm{d} t+z_{i}^{\pi}(t) P_{i}(t) \pi(t) \sigma(t) \mathrm{d} W(t) .
\end{aligned}
$$

Thus,

$$
\begin{aligned}
& \frac{1}{2} \mathrm{~d} z_{i}^{\pi}(t)^{2} P_{i}(t) \\
= & \frac{1}{2}\left\{2 z_{i}^{\pi}(t) P_{i}(t)\left[\gamma(t) \pi(t)^{\top}+\rho_{i} r(t)\right]+z_{i}^{\pi}(t)^{2} \beta(t) P_{i}(t)\right. \\
& \left.+P_{i}(t) \pi(t) \sigma(t) \sigma(t)^{\top} \pi(t)^{\top}\right\} \mathrm{d} t+z_{i}^{\pi}(t) P_{i}(t) \pi(t) \sigma(t) \mathrm{d} W(t) .
\end{aligned}
$$

Applying Itô formula to $z_{i}^{\pi}(t) g_{i}(t)$, it follows that

$$
\begin{aligned}
& \mathrm{d} z_{i}^{\pi}(t) g_{i}(t) \\
= & \left\{g_{i}(t) \gamma(t) \pi(t)^{\top}+g_{i}(t) \rho_{i} r(t)+z_{i}^{\pi}(t)\left[\beta(t) g_{i}(t)-\rho_{i} r(t) P_{i}(t)\right]\right\} \mathrm{d} t \\
& +g_{i}(t) \pi(t) \sigma(t) \mathrm{d} W(t) .
\end{aligned}
$$


We add the equations (3.7) and (3.8) together and integrate from $t_{i-1}$ to $t_{i}$. The left terms of equations (3.7) and (3.8) show that

$$
\begin{aligned}
& \mathbb{E}\left[\frac{P_{i}\left(t_{i}\right)}{2} z_{i}^{\pi}\left(t_{i}\right)^{2}-\frac{P_{i}\left(t_{i-1}\right)}{2} z_{i}^{\pi}\left(t_{i-1}\right)^{2}+z_{i}^{\pi}\left(t_{i}\right) g_{i}\left(t_{i}\right)-z_{i}^{\pi}\left(t_{i-1}\right) g_{i}\left(t_{i-1}\right)\right] \\
= & \mathbb{E}\left[\frac{\mu_{i}+P_{i+1}\left(t_{i}\right)}{2} z_{i}^{\pi}\left(t_{i}\right)^{2}-\frac{P_{i}\left(t_{i-1}\right)}{2} z_{i}^{\pi}\left(t_{i-1}\right)^{2}\right. \\
& \left.+z_{i}^{\pi}\left(t_{i}\right)\left[g_{i+1}\left(t_{i}\right)+P_{i+1}\left(t_{i}\right)\left(\rho_{i}-\rho_{i+1}\right)\right]-z_{i}^{\pi}\left(t_{i-1}\right) g_{i}\left(t_{i-1}\right)\right] \\
= & \mathbb{E}\left[\frac{\mu_{i}+P_{i+1}\left(t_{i}\right)}{2} z_{i}^{\pi}\left(t_{i}\right)^{2}-\frac{P_{i}\left(t_{i-1}\right)}{2}\left[z_{i-1}^{\pi}\left(t_{i-1}\right)+\rho_{i-1}-\rho_{i}\right]^{2}\right. \\
& \left.+z_{i}^{\pi}\left(t_{i}\right)\left[g_{i+1}\left(t_{i}\right)+P_{i+1}\left(t_{i}\right)\left(\rho_{i}-\rho_{i+1}\right)\right]-\left[z_{i-1}^{\pi}\left(t_{i-1}\right)+\rho_{i-1}-\rho_{i}\right] g_{i}\left(t_{i-1}\right)\right] \\
= & \mathbb{E}\left[\frac{\mu_{i}}{2} z_{i}^{\pi}\left(t_{i}\right)^{2}-\left(\rho_{i-1}-\rho_{i}\right)^{2} \frac{P_{i}\left(t_{i-1}\right)}{2}-\left(\rho_{i-1}-\rho_{i}\right) g_{i}\left(t_{i-1}\right)\right. \\
& +\frac{P_{i+1}\left(t_{i}\right)}{2} z_{i}^{\pi}\left(t_{i}\right)^{2}+P_{i+1}\left(t_{i}\right)\left(\rho_{i}-\rho_{i+1}\right) z_{i}^{\pi}\left(t_{i}\right)+z_{i}^{\pi}\left(t_{i}\right) g_{i+1}\left(t_{i}\right) \\
& \left.-\frac{P_{i}\left(t_{i-1}\right)}{2} z_{i-1}^{\pi}\left(t_{i-1}\right)^{2}-P_{i}\left(t_{i-1}\right)\left(\rho_{i-1}-\rho_{i}\right) z_{i-1}^{\pi}\left(t_{i-1}\right)-z_{i-1}^{\pi}\left(t_{i-1}\right) g_{i}\left(t_{i-1}\right)\right],
\end{aligned}
$$

where the second equality is derived by the following results,

$$
\begin{aligned}
z_{i}^{\pi}\left(t_{i-1}\right) & =Y^{\pi}\left(t_{i-1}\right)-\rho_{i} \\
& =Y^{\pi}\left(t_{i-1}\right)-\rho_{i-1}+\rho_{i-1}-\rho_{i} \\
& =z_{i-1}^{\pi}\left(t_{i-1}\right)+\rho_{i-1}-\rho_{i},
\end{aligned}
$$

where $z_{0}^{\pi}\left(t_{0}\right)=y, \rho_{0}=0$.

The right terms of equations (3.7) and (3.8) show that

$$
\begin{aligned}
& \mathbb{E}\left[\frac{P_{i}\left(t_{i}\right)}{2} z_{i}^{\pi}\left(t_{i}\right)^{2}-\frac{P_{i}\left(t_{i-1}\right)}{2} z_{i}^{\pi}\left(t_{i-1}\right)^{2}+z_{i}^{\pi}\left(t_{i}\right) g_{i}\left(t_{i}\right)-z_{i}^{\pi}\left(t_{i-1}\right) g_{i}\left(t_{i-1}\right)\right] \\
= & \frac{1}{2} \mathbb{E} \int_{t_{i-1}}^{t_{i}}\left\{P_{i}(t) \pi(t) \sigma(t) \sigma(t)^{\top} \pi(t)^{\top}+2 \gamma(t) \pi(t)^{\top}\left(z_{i}^{\pi}(t) P_{i}(t)+g_{i}(t)\right)\right. \\
& \left.+z_{i}^{\pi}(t)^{2} \beta(t) P_{i}(t)+2 z_{i}^{\pi}(t) \beta(t) g_{i}(t)+2 g_{i}(t) \rho_{i} r(t)\right\} \mathrm{d} t \\
= & \frac{1}{2} \mathbb{E} \int_{t_{i-1}}^{t_{i}}\left\{\left[\pi(t)+\gamma(t)\left(\sigma(t) \sigma(t)^{\top}\right)^{-1}\left(z_{i}^{\pi}(t)+\frac{g_{i}(t)}{P_{i}(t)}\right)\right] \sigma(t) P_{i}(t) \sigma(t)^{\top}\right. \\
& {\left[\pi(t)+\gamma(t)\left(\sigma(t) \sigma(t)^{\top}\right)^{-1}\left(z_{i}^{\pi}(t)+\frac{g_{i}(t)}{P_{i}(t)}\right]^{\top}\right.} \\
& \left.-\gamma(t)\left(P_{i}(t) \sigma(t) \sigma(t)^{\top}\right)^{-1} \gamma(t)^{\top} g_{i}(t)^{2}+2 g_{i}(t) \rho_{i} r(t)\right\} \mathrm{d} t .
\end{aligned}
$$


Thus, we have

$$
\begin{aligned}
& \mathbb{E}\left[\frac{\mu_{i}}{2} z_{i}^{\pi}\left(t_{i}\right)^{2}-\left(\rho_{i-1}-\rho_{i}\right)^{2} \frac{P_{i}\left(t_{i-1}\right)}{2}-\left(\rho_{i-1}-\rho_{i}\right) g_{i}\left(t_{i-1}\right)\right. \\
& +\frac{P_{i+1}\left(t_{i}\right)}{2} z_{i}^{\pi}\left(t_{i}\right)^{2}+P_{i+1}\left(t_{i}\right)\left(\rho_{i}-\rho_{i+1}\right) z_{i}^{\pi}\left(t_{i}\right)+z_{i}^{\pi}\left(t_{i}\right) g_{i+1}\left(t_{i}\right) \\
& \left.-\frac{P_{i}\left(t_{i-1}\right)}{2} z_{i-1}^{\pi}\left(t_{i-1}\right)^{2}-P_{i}\left(t_{i-1}\right)\left(\rho_{i-1}-\rho_{i}\right) z_{i-1}^{\pi}\left(t_{i-1}\right)-z_{i-1}^{\pi}\left(t_{i-1}\right) g_{i}\left(t_{i-1}\right)\right] \\
= & \frac{1}{2} \mathbb{E} \int_{t_{i-1}}^{t_{i}}\left\{\left[\pi(t)+\gamma(t)\left(\sigma(t) \sigma(t)^{\top}\right)^{-1}\left(z_{i}^{\pi}(t)+\frac{g_{i}(t)}{P_{i}(t)}\right)\right] \sigma(t) P_{i}(t) \sigma(t)^{\top}\right. \\
& {\left[\pi(t)+\gamma(t)\left(\sigma(t) \sigma(t)^{\top}\right)^{-1}\left(z_{i}^{\pi}(t)+\frac{g_{i}(t)}{P_{i}(t)}\right)\right]^{\top} } \\
& \left.-\gamma(t)\left(P_{i}(t) \sigma(t) \sigma(t)^{\top}\right)^{-1} \gamma(t)^{\top} g_{i}(t)^{2}+2 g_{i}(t) \rho_{i} r(t)\right\} \mathrm{d} t .
\end{aligned}
$$

Adding $i$ on both sides of equation (3.9) from 1 to $N$, it follows that

$$
\begin{aligned}
& \sum_{i=1}^{N} \mathbb{E}\left[\frac{\mu_{i}}{2} z_{i}^{\pi}\left(t_{i}\right)^{2}-\left(\rho_{i-1}-\rho_{i}\right)^{2} \frac{P_{i}\left(t_{i-1}\right)}{2}-\left(\rho_{i-1}-\rho_{i}\right) g_{i}\left(t_{i-1}\right)\right. \\
& +\frac{P_{i+1}\left(t_{i}\right)}{2} z_{i}^{\pi}\left(t_{i}\right)^{2}+P_{i+1}\left(t_{i}\right)\left(\rho_{i}-\rho_{i+1}\right) z_{i}^{\pi}\left(t_{i}\right)+z_{i}^{\pi}\left(t_{i}\right) g_{i+1}\left(t_{i}\right) \\
& \left.-\frac{P_{i}\left(t_{i-1}\right)}{2} z_{i-1}^{\pi}\left(t_{i-1}\right)^{2}-P_{i}\left(t_{i-1}\right)\left(\rho_{i-1}-\rho_{i}\right) z_{i-1}^{\pi}\left(t_{i-1}\right)-z_{i-1}^{\pi}\left(t_{i-1}\right) g_{i}\left(t_{i-1}\right)\right] \\
= & \sum_{i=1}^{N} \mathbb{E}\left[\frac{\mu_{i}}{2} z_{i}^{\pi}\left(t_{i}\right)^{2}-\left(\rho_{i-1}-\rho_{i}\right)^{2} \frac{P_{i}\left(t_{i-1}\right)}{2}-\left(\rho_{i-1}-\rho_{i}\right) g_{i}\left(t_{i-1}\right)\right] \\
& -\mathbb{E}\left[\frac{P_{1}\left(t_{0}\right)}{2} z_{0}^{\pi}\left(t_{0}\right)^{2}+P_{1}\left(t_{0}\right)\left(\rho_{0}-\rho_{1}\right) z_{0}^{\pi}\left(t_{0}\right)+z_{0}^{\pi}\left(t_{0}\right) g_{1}\left(t_{0}\right)\right] \\
= & \sum_{i=1}^{N} \frac{1}{2} \mathbb{E} \int_{t_{i-1}}^{t_{i}}\left\{\left[\pi(t)+\gamma(t)\left(\sigma(t) \sigma(t)^{\top}\right)^{-1}\left(z_{i}^{\pi}(t)+\frac{g_{i}(t)}{P_{i}(t)}\right)\right] \sigma(t) P_{i}(t) \sigma(t)^{\top}\right. \\
& {\left[\pi(t)+\gamma(t)\left(\sigma(t) \sigma(t)^{\top}\right)^{-1}\left(z_{i}^{\pi}(t)+\frac{g_{i}(t)}{P_{i}(t)}\right)\right]^{\top} } \\
& \left.-\gamma(t)\left(P_{i}(t) \sigma(t) \sigma(t)^{\top}\right)^{-1} \gamma(t)^{\top} g_{i}(t)^{2}+2 g_{i}(t) \rho_{i} r(t)\right\} \mathrm{d} t,
\end{aligned}
$$

and thus

$$
\begin{aligned}
& \mathbb{E}\left[\sum_{i=1}^{N} \frac{\mu_{i}}{2} z_{i}^{\pi}\left(t_{i}\right)^{2}\right] \\
= & \sum_{i=1}^{N} \frac{1}{2} \mathbb{E} \int_{t_{i-1}}^{t_{i}}\left\{\left[\pi(t)+\gamma(t)\left(\sigma(t) \sigma(t)^{\top}\right)^{-1}\left(z_{i}^{\pi}(t)+\frac{g_{i}(t)}{P_{i}(t)}\right)\right] \sigma(t) \sigma(t)^{\top}\right. \\
& {\left[\pi(t)+\gamma(t)\left(\sigma(t) \sigma(t)^{\top}\right)^{-1}\left(z_{i}^{\pi}(t)+\frac{g_{i}(t)}{P_{i}(t)}\right)\right]^{\top} } \\
& \left.-\gamma(t)\left(P_{i}(t) \sigma(t) \sigma(t)^{\top}\right)^{-1} \gamma(t)^{\top} g_{i}(t)^{2}+2 g_{i}(t) \rho_{i} r(t)\right\} \mathrm{d} t \\
& +\sum_{i=1}^{N} \mathbb{E}\left[\left(\rho_{i-1}-\rho_{i}\right)^{2} \frac{P_{i}\left(t_{i-1}\right)}{2}+\left(\rho_{i-1}-\rho_{i}\right) g_{i}\left(t_{i-1}\right)\right] \\
& +\mathbb{E}\left[\frac{P_{1}\left(t_{0}\right)}{2} z_{0}^{\pi}\left(t_{0}\right)^{2}+P_{1}\left(t_{0}\right)\left(\rho_{0}-\rho_{1}\right) z_{0}^{\pi}\left(t_{0}\right)+z_{0}^{\pi}\left(t_{0}\right) g_{1}\left(t_{0}\right)\right] .
\end{aligned}
$$


Based on the representation of $\mathbb{E}\left[\sum_{i=1}^{N} \frac{\mu_{i}}{2} z_{i}^{\pi}\left(t_{i}\right)^{2}\right]$, we can obtain an optimal strategy $\pi^{*}(\cdot)$ for $J_{4}(\pi(\cdot))$, for $t \in\left(t_{i-1}, t_{i}\right], i=1,2, \ldots, N$,

$$
\pi^{*}(t)=-\gamma(t)\left(\sigma(t) \sigma(t)^{\top}\right)^{-1}\left(z_{i}^{\pi^{*}}(t)+\frac{g_{i}(t)}{P_{i}(t)}\right)
$$

Note that

$$
\frac{g_{i}(t)}{P_{i}(t)}=\frac{g_{i}\left(t_{i}\right)}{P_{i}\left(t_{i}\right)} e^{-\int_{t}^{t_{i}} r(s) \mathrm{d} s}+\rho_{i}\left(1-e^{-\int_{t}^{t_{i}} r(s) \mathrm{d} s}\right), t_{i-1}<t \leq t_{i},
$$

where

$$
\frac{g_{i}\left(t_{i}\right)}{P_{i}\left(t_{i}\right)}=\frac{g_{i+1}\left(t_{i}\right)+P_{i+1}\left(t_{i}\right)\left(\rho_{i}-\rho_{i+1}\right)}{\mu_{i}+P_{i+1}\left(t_{i}\right)}, i=1,2, \ldots, N,
$$

which leads to

$$
\pi^{*}(t)=\gamma(t)\left(\sigma(t) \sigma(t)^{\top}\right)^{-1}\left[\left(\rho_{i}-\frac{g_{i}\left(t_{i}\right)}{P_{i}\left(t_{i}\right)}\right) e^{-\int_{t}^{t_{i}} r(s) \mathrm{d} s}-Y^{*}(t)\right], t_{i-1}<t \leq t_{i},
$$

where $Y^{*}(t)=z^{\pi^{*}}(t)+\rho_{i}, t_{i-1}<t \leq t_{i}$ and $i=1,2, \ldots, N$.

This completes the proof.

Now, we consider the process of portfolio asset equation according to $\pi^{*}(\cdot)$,

$$
\left\{\begin{aligned}
\mathrm{d} Y^{*}(t) & =\left[r(t) Y^{*}(t)+\gamma(t) \pi^{*}(t)^{\top}\right] \mathrm{d} t+\pi^{*}(t) \sigma(t) \mathrm{d} W(t), \\
Y^{*}(0) & =y
\end{aligned}\right.
$$

Remark 3.3. Let Assumptions $\mathbf{H}_{1}$ and $\mathbf{H}_{2}$ hold, putting $\pi^{*}(\cdot)$ into equation (3.12). Note that, $\pi^{*}(\cdot)$ is a linear function of $Y^{*}(\cdot)$. Thus, equation (3.12) admits a unique solution.

$\mathbb{E}\left[Y^{*}(\cdot)\right]$ and $\mathbb{E}\left[Y^{*}(\cdot)^{2}\right]$ satisfy the following linear ordinary differential equations:

$$
\left\{\begin{aligned}
\mathrm{d} \mathbb{E}\left[Y^{*}(t)\right] & =\left[(r(t)-\beta(t)) \mathbb{E}\left[Y^{*}(t)\right]+\left(\rho_{i}-\frac{g_{i}\left(t_{i}\right)}{P_{i}\left(t_{i}\right)}\right) e^{-\int_{t}^{t_{i}} r(s) \mathrm{d} s} \beta(t)\right] \mathrm{d} t, \\
Y^{*}(0) & =y, t_{i-1}<t \leq t_{i}, i=1,2, \ldots, N
\end{aligned}\right.
$$

and

$$
\left\{\begin{aligned}
\mathrm{d} \mathbb{E}\left[Y^{*}(t)^{2}\right] & =\left[(2 r(t)-\beta(t)) \mathbb{E}\left[Y^{*}(t)^{2}\right]+\left(\rho_{i}-\frac{g_{i}\left(t_{i}\right)}{P_{i}\left(t_{i}\right)}\right)^{2} e^{-\int_{t}^{t_{i}} 2 r(s) \mathrm{d} s} \beta(t)\right] \mathrm{d} t, \\
Y^{*}(0)^{2} & =y^{2}, t_{i-1}<t \leq t_{i}, \quad i=1,2, \ldots, N
\end{aligned}\right.
$$

In the following, we investigate the efficient frontier of the multi-time state mean-variance $\operatorname{Var}\left(Y^{*}\left(t_{i}\right)\right)$ and $\mathbb{E}\left[Y^{*}\left(t_{i}\right)\right], i=1,2, \ldots, N$. 
Lemma 3.4. Let Assumptions $\boldsymbol{H}_{1}$ and $\boldsymbol{H}_{2}$ hold, the relationship of $\operatorname{Var}\left(Y^{*}\left(t_{i}\right)\right)$ and $\mathbb{E}\left[Y^{*}\left(t_{i}\right)\right]$ is given as follows:

$$
\operatorname{Var}\left(Y^{*}\left(t_{i}\right)\right)=\operatorname{Var}\left(Y^{*}\left(t_{i-1}\right)\right) e^{\int_{t_{i-1}}^{t_{i}}[2 r(t)-\beta(t)] \mathrm{d} t}+\frac{\left(\mathbb{E}\left[Y^{*}\left(t_{i}\right)\right]-\mathbb{E}\left[Y^{*}\left(t_{i-1}\right)\right] e^{\int_{t_{i-1}}^{t_{i}} r(t) \mathrm{d} t}\right)^{2}}{e^{\int_{t_{i-1}}^{t_{i}} \beta(t) \mathrm{d} t}-1},
$$

where $i=1,2, \ldots, N$.

Proof. Combining equations (3.13) and (3.14), we have for $i=1,2, \ldots, N$,

$$
\mathbb{E}\left[Y^{*}\left(t_{i}\right)\right]=\mathbb{E}\left[Y^{*}\left(t_{i-1}\right)\right] e^{\int_{t_{i-1}}^{t_{i}}[r(t)-\beta(t)] \mathrm{d} t}+\left(\rho_{i}-\frac{g_{i}\left(t_{i}\right)}{P_{i}\left(t_{i}\right)}\right)\left(1-e^{-\int_{t_{i-1}}^{t_{i}} \beta(t) \mathrm{d} t}\right),
$$

and

$$
\mathbb{E}\left[Y^{*}\left(t_{i}\right)^{2}\right]=\mathbb{E}\left[Y^{*}\left(t_{i-1}\right)^{2}\right] e^{\int_{t_{i-1}}^{t_{i}}[2 r(t)-\beta(t)] \mathrm{d} t}+\left(\rho_{i}-\frac{g_{i}\left(t_{i}\right)}{P_{i}\left(t_{i}\right)}\right)^{2}\left(1-e^{-\int_{t_{i-1}}^{t_{i}} \beta(t) \mathrm{d} t}\right) .
$$

By equation (3.16), we have

$$
\rho_{i}-\frac{g_{i}\left(t_{i}\right)}{P_{i}\left(t_{i}\right)}=\frac{\mathbb{E}\left[Y^{*}\left(t_{i}\right)\right]-\mathbb{E}\left[Y^{*}\left(t_{i-1}\right)\right] e^{\int_{t_{i-1}}^{t_{i}}[r(t)-\beta(t)] \mathrm{d} t}}{1-e^{-\int_{t_{i-1}}^{t_{i}} \beta(t) \mathrm{d} t}} .
$$

Plugging $\rho_{i}-\frac{g_{i}\left(t_{i}\right)}{P_{i}\left(t_{i}\right)}$ into equation (3.17), it follows that

$$
\mathbb{E}\left[Y^{*}\left(t_{i}\right)^{2}\right]=\mathbb{E}\left[Y^{*}\left(t_{i-1}\right)^{2}\right] e^{\int_{t_{i-1}}^{t_{i}}[2 r(t)-\beta(t)] \mathrm{d} t}+\frac{\left(\mathbb{E}\left[Y^{*}\left(t_{i}\right)\right]-\mathbb{E}\left[Y^{*}\left(t_{i-1}\right)\right] e^{\int_{t_{i-1}}^{t_{i}}[r(t)-\beta(t)] \mathrm{d} t}\right)^{2}}{1-e^{-\int_{t_{i-1}}^{t_{i}} \beta(t) \mathrm{d} t}}
$$

and thus

$$
\begin{aligned}
& \operatorname{Var}\left(Y^{*}\left(t_{i}\right)\right)\left(1-e^{-\int_{t_{i-1}}^{t_{i}} \beta(t) \mathrm{d} t}\right) \\
= & \left(\mathbb{E}\left[Y^{*}\left(t_{i-1}\right)^{2}\right]-\left[\mathbb{E} Y^{*}\left(t_{i-1}\right)\right]^{2}\right) e^{\int_{t_{i-1}}^{t_{i}}[2 r(t)-\beta(t)] \mathrm{d} t}\left(1-e^{-\int_{t_{i-1}}^{t_{i}} \beta(t) \mathrm{d} t}\right) \\
& +\left[\mathbb{E} Y^{*}\left(t_{i-1}\right)\right]^{2}\left(e^{\int_{t_{i-1}}^{t_{i}}[2 r(t)-\beta(t)] \mathrm{d} t}-e^{\int_{t_{i-1}}^{t_{i}}[2 r(t)-2 \beta(t)] \mathrm{d} t}\right) \\
& +\left(\mathbb{E}\left[Y^{*}\left(t_{i}\right)\right]-\mathbb{E}\left[Y^{*}\left(t_{i-1}\right)\right] e^{\int_{t_{i-1}}^{t_{i}}[r(t)-\beta(t)] \mathrm{d} t}\right)^{2}+\left(e^{-\int_{t_{i-1}}^{t_{i}} \beta(s) \mathrm{d} s}-1\right)\left[\mathbb{E} Y^{*}\left(t_{i}\right)\right]^{2} \\
= & \left(\mathbb{E}\left[Y^{*}\left(t_{i-1}\right)^{2}\right]-\left[\mathbb{E} Y^{*}\left(t_{i-1}\right)\right]^{2}\right) e^{\int_{t_{i-1}}^{t_{i}}[2 r(t)-\beta(t)] \mathrm{d} t}\left(1-e^{-\int_{t_{i-1}}^{t_{i}} \beta(t) \mathrm{d} t}\right) \\
& +\left[\mathbb{E} Y^{*}\left(t_{i-1}\right)\right]^{2} e^{\int_{t_{i-1}}^{t_{i}}[2 r(t)-\beta(t)] \mathrm{d} t}+\left[\mathbb{E} Y^{*}\left(t_{i}\right)\right]^{2} e^{-\int_{t_{i-1}}^{t_{i}} \beta(t) \mathrm{d} t} \\
& -2 \mathbb{E}\left[Y^{*}\left(t_{i}\right)\right] \mathbb{E}\left[Y^{*}\left(t_{i-1}\right)\right] e^{\int_{t_{i-1}}^{t_{i}}[r(t)-\beta(t)] \mathrm{d} t} .
\end{aligned}
$$


Note that

$$
\begin{aligned}
& {\left[\mathbb{E} Y^{*}\left(t_{i-1}\right)\right]^{2} e^{\int_{t_{i-1}}^{t_{i}}[2 r(t)-\beta(t)] \mathrm{d} t}+\left[\mathbb{E} Y^{*}\left(t_{i}\right)\right]^{2} e^{-\int_{t_{i-1}}^{t_{i}} \beta(t) \mathrm{d} t} } \\
& -2 \mathbb{E}\left[Y^{*}\left(t_{i}\right)\right] \mathbb{E}\left[Y^{*}\left(t_{i-1}\right)\right] e^{\int_{t_{i-1}}^{t_{i}}[r(t)-\beta(t)] \mathrm{d} t} \\
= & e^{-\int_{t_{i-1}}^{t_{i}} \beta(t) \mathrm{d} t}\left(\mathbb{E}\left[Y^{*}\left(t_{i}\right)\right]-\mathbb{E}\left[Y^{*}\left(t_{i-1}\right)\right] e^{\int_{t_{i-1}}^{t_{i}} r(t) \mathrm{d} t}\right)^{2}
\end{aligned}
$$

Thus, we have

$$
\begin{aligned}
& \operatorname{Var}\left(Y^{*}\left(t_{i}\right)\right)\left(1-e^{-\int_{t_{i-1}}^{t_{i}} \beta(t) \mathrm{d} t}\right) \\
= & \left(\mathbb{E}\left[Y^{*}\left(t_{i-1}\right)^{2}\right]-\left[\mathbb{E} Y^{*}\left(t_{i-1}\right)\right]^{2}\right) e^{\int_{t_{i-1}}^{t_{i}}[2 r(t)-\beta(t)] \mathrm{d} t}\left(1-e^{-\int_{t_{i-1}}^{t_{i}} \beta(t) \mathrm{d} t}\right) \\
& +e^{-\int_{t_{i-1}}^{t_{i}} \beta(t) \mathrm{d} t}\left(\mathbb{E}\left[Y^{*}\left(t_{i}\right)\right]-\mathbb{E}\left[Y^{*}\left(t_{i-1}\right)\right] e^{\int_{t_{i-1}}^{t_{i}} r(t) \mathrm{d} t}\right)^{2},
\end{aligned}
$$

which deduces that

$$
\operatorname{Var}\left(Y^{*}\left(t_{i}\right)\right)=\operatorname{Var}\left(Y^{*}\left(t_{i-1}\right)\right) e^{\int_{t_{i-1}}^{t_{i}}[2 r(t)-\beta(t)] \mathrm{d} t}+\frac{\left(\mathbb{E}\left[Y^{*}\left(t_{i}\right)\right]-\mathbb{E}\left[Y^{*}\left(t_{i-1}\right)\right] e^{\int_{t_{i-1}}^{t_{i}} r(t) \mathrm{d} t}\right)^{2}}{e^{\int_{t_{i-1}}^{t_{i}} \beta(t) \mathrm{d} t}-1} .
$$

This completes the proof.

Remark 3.5. Specially, for $i=1$, one obtains

$$
\operatorname{Var}\left(Y^{*}\left(t_{1}\right)\right)=\frac{\left(\mathbb{E}\left[Y^{*}\left(t_{1}\right)\right]-y e^{\int_{t_{0}}^{t_{1}} r(t) \mathrm{d} t}\right)^{2}}{e^{\int_{t_{0}}^{t_{1}} \beta(t) \mathrm{d} t}-1},
$$

which is the same as the efficient frontier in [33].

It should be noted that the optimal strategy $\pi^{*}(\cdot)$ of cost functional (3.3) depends on the parameters $\mu=$ $\left(\mu_{1}, \ldots, \mu_{N}\right), \lambda=\left(\lambda_{1}, \ldots, \lambda_{N}\right) \in \mathbb{R}^{N}$. We want to show that there exist $\lambda$ and $\mu$ such that the optimal strategy $\pi^{*}(\cdot)$ of cost functional (3.3) is an optimal strategy of cost functional (3.2).

Theorem 3.6. Let Assumptions $\boldsymbol{H}_{1}, \boldsymbol{H}_{2}$ hold, and

$$
\begin{aligned}
& L_{i}-L_{i-1} e^{\int_{t_{i-1}}^{t_{i}} r(t) \mathrm{d} t} \neq 0, \quad i=1,2, \ldots, N ; \\
& {\left[1+P_{i+1}\left(t_{i}\right) \rho_{i+1}-g_{i+1}\left(t_{i}\right)\right]\left(1-e^{-\int_{t_{i-1}}^{t_{i}} \beta(t) \mathrm{d} t}\right) } \\
\neq \quad & {\left[L_{i}-L_{i-1} e^{\int_{t_{i-1}}^{t_{i}}[r(t)-\beta(t)] \mathrm{d} t}\right] P_{i+1}\left(t_{i}\right), \quad i=1,2, \ldots, N-1, }
\end{aligned}
$$

where $L_{0}=y$. There exists $\lambda^{*}=\left(\lambda_{1}^{*}, \lambda_{2}^{*}, \ldots, \lambda_{N}^{*}\right), \mu=\left(\mu_{1}, \mu_{2}, \ldots, \mu_{N}\right) \in \mathbb{R}^{N}$ which are determined by

$$
\lambda_{i}^{*}=1+\mu_{i} \mathbb{E}\left[Y^{*}\left(t_{i}\right)\right], \rho_{i}=\frac{\lambda_{i}^{*}}{\mu_{i}}, i=1,2, \ldots, N,
$$


such that the optimal strategy $\pi^{*}(\cdot)$ of cost functional (3.3) is an optimal strategy of cost functional (3.2).

Proof. By Theorem 3.2, an optimal strategy of model (3.2) can be solved by (3.3), let

$$
\lambda_{i}^{*}=1+\mu_{i} \mathbb{E}\left[Y^{*}\left(t_{i}\right)\right], \rho_{i}=\frac{\lambda_{i}^{*}}{\mu_{i}}, i=1,2, \ldots, N .
$$

Note that $\mathbb{E}\left[Y^{*}\left(t_{i}\right)\right]$ depends on $\lambda_{i}^{*}$. To solve the parameters $\lambda_{i}^{*}, i=1,2, \ldots, N$, by equation (3.16), we first consider the case $i=N$,

$$
\mathbb{E}\left[Y^{*}\left(t_{N}\right)\right]=\mathbb{E}\left[Y^{*}\left(t_{N-1}\right)\right] e^{\int_{t_{N-1}}^{t_{N}}[r(t)-\beta(t)] \mathrm{d} t}+\frac{\lambda_{N}^{*}}{\mu_{N}}\left[1-e^{-\int_{t_{N-1}}^{t_{N}} \beta(t) \mathrm{d} t}\right]
$$

and

$$
\lambda_{N}^{*}=1+\mu_{N} \mathbb{E}\left[Y^{*}\left(t_{N-1}\right)\right] e^{\int_{t_{N-1}}^{t_{N}}[r(t)-\beta(t)] \mathrm{d} t}+\lambda_{N}^{*}\left[1-e^{-\int_{t_{N-1}}^{t_{N}} \beta(t) \mathrm{d} t}\right] .
$$

Thus, we have

$$
\lambda_{N}^{*}=e^{\int_{t_{N-1}}^{t_{N}} \beta(t) \mathrm{d} t}+\mu_{N} \mathbb{E}\left[Y^{*}\left(t_{N-1}\right)\right] e^{\int_{t_{N-1}}^{t_{N}} r(t) \mathrm{d} t} .
$$

Based on the representation of $\lambda_{N}^{*}$, by equation (3.21), we have

$$
\mathbb{E}\left[Y^{*}\left(t_{N}\right)\right]=\frac{e^{\int_{t_{N-1}}^{t_{N}} \beta(t) \mathrm{d} t}-1}{\mu_{N}}+\mathbb{E}\left[Y^{*}\left(t_{N-1}\right)\right] e^{\int_{t_{N-1}}^{t_{N}} r(t) \mathrm{d} t},
$$

which indicates that

$$
\mu_{N}=\frac{e^{\int_{t_{N-1}}^{t_{N}} \beta(t) \mathrm{d} t}-1}{\mathbb{E}\left[Y^{*}\left(t_{N}\right)\right]-\mathbb{E}\left[Y^{*}\left(t_{N-1}\right)\right] e^{\int_{t_{N-1}}^{t_{N}} r(t) \mathrm{d} t}} .
$$

Based on constrained condition (2.3) of $\mathbb{E}\left[Y^{*}\left(t_{N}\right)\right]=L_{N}, \mathbb{E}\left[Y^{*}\left(t_{N-1}\right)\right]=L_{N-1}$ and condition (3.18), we can solve $\lambda_{N}^{*}$ and $\mu_{N} \neq 0$.

In the following, we consider the case $i=N-1$. By equations (3.16) and (3.20), one obtains

$$
\lambda_{N-1}^{*}=1+\mu_{N-1} \mathbb{E}\left[Y^{*}\left(t_{N-1}\right)\right],
$$

and

$$
\begin{aligned}
& \mathbb{E}\left[Y^{*}\left(t_{N-1}\right)\right]-\mathbb{E}\left[Y^{*}\left(t_{N-2}\right)\right] e^{\int_{t_{N-2}}^{t_{N-1}}[r(t)-\beta(t)] \mathrm{d} t} \\
= & \left(\rho_{N-1}-\frac{g_{N-1}\left(t_{N-1}\right)}{P_{N-1}\left(t_{N-1}\right)}\right)\left[1-e^{-\int_{t_{N-2}}^{t_{N-1}} \beta(t) \mathrm{d} t}\right] \\
= & \left(\frac{\lambda_{N-1}^{*}}{\mu_{N-1}}-\frac{g_{N}\left(t_{N-1}\right)+P_{N}\left(t_{N-1}\right)\left(\rho_{N-1}-\rho_{N}\right)}{\mu_{N-1}+P_{N}\left(t_{N-1}\right)}\right)\left[1-e^{-\int_{t_{N-2}}^{t_{N-1}} \beta(t) \mathrm{d} t}\right] \\
= & \frac{\lambda_{N-1}^{*}+P_{N}\left(t_{N-1}\right) \rho_{N}-g_{N}\left(t_{N-1}\right)}{\mu_{N-1}+P_{N}\left(t_{N-1}\right)}\left[1-e^{-\int_{t_{N-2}}^{t_{N-1}} \beta(t) \mathrm{d} t}\right],
\end{aligned}
$$


and thus

$$
\begin{aligned}
& \mathbb{E}\left[Y^{*}\left(t_{N-1}\right)\right]-\mathbb{E}\left[Y^{*}\left(t_{N-2}\right)\right] e^{\int_{t_{N-2}}^{t_{N-1}}[r(t)-\beta(t)] \mathrm{d} t} \\
& =\frac{\lambda_{N-1}^{*}+P_{N}\left(t_{N-1}\right) \rho_{N}-g_{N}\left(t_{N-1}\right)}{\mu_{N-1}+P_{N}\left(t_{N-1}\right)}\left[1-e^{-\int_{t_{N-2}}^{t_{N-1}} \beta(t) \mathrm{d} t}\right] .
\end{aligned}
$$

It follows that

$$
\begin{aligned}
\lambda_{N-1}^{*}= & 1+\mu_{N-1} \mathbb{E}\left[Y^{*}\left(t_{N-1}\right)\right] \\
= & 1+\frac{P_{N}\left(t_{N-1}\right) \rho_{N}-g_{N}\left(t_{N-1}\right)}{u_{N-1}+P_{N}\left(t_{N-1}\right)}\left[1-e^{-\int_{t_{N-2}}^{t_{N-1}} \beta(t) \mathrm{d} t}\right] \mu_{N-1} \\
& +\mu_{N-1} \mathbb{E}\left[Y^{*}\left(t_{N-2}\right)\right] e^{\int_{t_{N-2}}^{t_{N-1}}[r(t)-\beta(t)] \mathrm{d} t}+\frac{\mu_{N-1} \lambda_{N-1}^{*}}{u_{N-1}+P_{N}\left(t_{N-1}\right)}\left[1-e^{-\int_{t_{N-2}}^{t_{N-1}} \beta(t) \mathrm{d} t}\right] .
\end{aligned}
$$

Note that the coefficient of $\lambda_{N-1}^{*}$ is

$$
I_{N-1}^{*}=\frac{P_{N}\left(t_{N-1}\right)+\mu_{N-1} e^{-\int_{t_{N-2}}^{t_{N-1}} \beta(t) \mathrm{d} t}}{\mu_{N-1}+P_{N}\left(t_{N-1}\right)}>0,
$$

which indicates that there exists a unique solution for $\lambda_{N-1}^{*}$,

$$
\begin{aligned}
\lambda_{N-1}^{*}= & \frac{P_{N}\left(t_{N-1}\right)+\mu_{N-1}}{P_{N}\left(t_{N-1}\right)+\mu_{N-1} e^{-\int_{t_{N-2}}^{t_{N-1}} \beta(t) \mathrm{d} t}} \\
& +\mu_{N-1}\left(\frac{P_{N}\left(t_{N-1}\right) \rho_{N}-g_{N}\left(t_{N-1}\right)}{P_{N}\left(t_{N-1}\right)+\mu_{N-1} e^{-\int_{t_{N-2}}^{t_{N-1}} \beta(t) \mathrm{d} t}\left[1-e^{-\int_{t_{N-2}}^{t_{N}-1} \beta(t) \mathrm{d} t}\right]}\right. \\
& \left.+\frac{P_{N}\left(t_{N-1}\right)+u_{N-1}}{P_{N}\left(t_{N-1}\right)+\mu_{N-1} e^{-\int_{t_{N-2}}^{t_{N-1}} \beta(t) \mathrm{d} t}} \mathbb{E}\left[Y^{*}\left(t_{N-2}\right)\right] e^{\int_{t_{N-2}}^{t_{N-1}}[r(t)-\beta(t)] \mathrm{d} t}\right) .
\end{aligned}
$$

Combining equations (3.24) and (3.25), we have,

$$
\begin{aligned}
\mu_{N-1}= & \frac{\left[1+P_{N}\left(t_{N-1}\right) \rho_{N}-g_{N}\left(t_{N-1}\right)\right]\left(e^{\int_{t_{N-2}}^{t_{N-1}} \beta(t) \mathrm{d} t}-1\right)}{\mathbb{E}\left[Y^{*}\left(t_{N-1}\right)\right]-\mathbb{E}\left[Y^{*}\left(t_{N-2}\right)\right] e^{\int_{t_{N-2}}^{t_{N-1}} r(t) \mathrm{d} t}} \\
& -\frac{\left[\mathbb{E}\left[Y^{*}\left(t_{N-1}\right)\right]-\mathbb{E}\left[Y^{*}\left(t_{N-2}\right)\right] e^{\int_{N-2}^{t_{N-1}}[r(t)-\beta(t)] \mathrm{d} t}\right] P_{N}\left(t_{N-1}\right) e^{\int_{t_{N-2}}^{t_{N-1}} \beta(t) \mathrm{d} t}}{\mathbb{E}\left[Y^{*}\left(t_{N-1}\right)\right]-\mathbb{E}\left[Y^{*}\left(t_{N-2}\right)\right] e^{\int_{t_{N-2}}^{t} r(t) \mathrm{d} t}} .
\end{aligned}
$$

Again, based on constrained condition (2.3) of $\mathbb{E}\left[Y^{*}\left(t_{N-1}\right)\right]=L_{N-1}, \mathbb{E}\left[Y^{*}\left(t_{N-2}\right)\right]=L_{N-2}$ and condition $(3.18)$, we can solve $\lambda_{N-1}^{*}$ and $\mu_{N-1} \neq 0$. 
Similar to the case $i=N-1$, we can solve $\lambda_{i}^{*}, \mu_{i}, i=1,2, \ldots, N-1$ step by step from $N-1$ to 1 ,

$$
\begin{aligned}
\lambda_{i}^{*}= & \frac{P_{i+1}\left(t_{i}\right)+\mu_{i}}{P_{i+1}\left(t_{i}\right)+\mu_{i} e^{-\int_{t_{i-1}}^{t_{i}} \beta(t) \mathrm{d} t}} \\
& +\mu_{i}\left(\frac{P_{i+1}\left(t_{i}\right) \rho_{i+1}-g_{i+1}\left(t_{i}\right)}{P_{i+1}\left(t_{i}\right)+\mu_{i} e^{-\int_{t_{i-1}}^{t_{i}} \beta(t) \mathrm{d} t}}\left[1-e^{-\int_{t_{i-1}}^{t_{i}} \beta(t) \mathrm{d} t}\right]\right. \\
& \left.+\frac{P_{i+1}\left(t_{i}\right)+u_{i}}{P_{i+1}\left(t_{i}\right)+\mu_{i} e^{-\int_{t_{i-1}}^{t_{i}} \beta(t) \mathrm{d} t}} \mathbb{E}\left[Y^{*}\left(t_{i-1}\right)\right] e^{\int_{t_{i-1}}^{t_{i}}[r(t)-\beta(t)] \mathrm{d} t}\right),
\end{aligned}
$$

and

$$
\begin{aligned}
\mu_{i}= & \frac{\left[1+P_{i+1}\left(t_{i}\right) \rho_{i+1}-g_{i+1}\left(t_{i}\right)\right]\left(e^{\int_{t_{i-1}}^{t_{i}} \beta(t) \mathrm{d} t}-1\right)}{\mathbb{E}\left[Y^{*}\left(t_{i}\right)\right]-\mathbb{E}\left[Y^{*}\left(t_{i-1}\right)\right] e^{\int_{t_{i-1}}^{t_{i}} r(t) \mathrm{d} t}} \\
& -\frac{\left[\mathbb{E}\left[Y^{*}\left(t_{i}\right)\right]-\mathbb{E}\left[Y^{*}\left(t_{i-1}\right)\right] e^{\int_{t_{i-1}}^{t_{i}}[r(t)-\beta(t)] \mathrm{d} t}\right] P_{i+1}\left(t_{i}\right) e^{\int_{t_{i-1}}^{t_{i}} \beta(t) \mathrm{d} t}}{\mathbb{E}\left[Y^{*}\left(t_{i}\right)\right]-\mathbb{E}\left[Y^{*}\left(t_{i-1}\right)\right] e^{\int_{t_{i-1}}^{t_{i}} r(t) \mathrm{d} t}} .
\end{aligned}
$$

Therefore, the optimal strategy $\pi^{*}(\cdot)$ of cost functional (3.3) is an optimal strategy of cost functional (3.2). This completes the proof.

Remark 3.7. The conditions (3.18) and (3.20) guarantee that cost functional (3.2) has an optimal strategy with the parameters $\lambda^{*}$ and $\mu$. However, we have not given the condition for $L_{i}, i=1, \ldots, N$ to guarantee $\mu_{i} \neq 0, i=1,2, \ldots, N$ which satisfies condition (3.18). In the following section, we give the condition for $L_{1}, L_{2}$ to guarantee $\mu_{1}, \mu_{2} \neq 0$ for the case $N=2$ and solve $\lambda^{*}=\left(\lambda_{1}^{*}, \lambda_{2}^{*}\right), \mu=\left(\mu_{1}, \mu_{2}\right)$ by $\mathbb{E}\left[Y^{\pi}\left(t_{1}\right)\right]=L_{1}, \mathbb{E}\left[Y^{\pi}\left(t_{2}\right)\right]=$ $L_{2}$.

\section{ExPLICIT SOLUTION AND SIMULATION}

In this section, we consider a simple example with $N=2$ which is used to verify the results in Theorem 3.6 and investigate an explicit solution for the parameters $\lambda^{*}=\left(\lambda_{1}, \lambda_{2}\right), \mu=\left(\mu_{1}, \mu_{2}\right),\left(\mathbb{E}\left[Y^{*}\left(t_{1}\right)\right], \mathbb{E}\left[Y^{*}\left(t_{2}\right)\right]\right)$, and variance $\left(\operatorname{Var}\left[Y^{*}\left(t_{1}\right)\right], \operatorname{Var}\left[Y^{*}\left(t_{2}\right)\right]\right)$. Furthermore, we compare our multi-time state mean-variance model with the classical mean-variance model.

\subsection{Explicit solution}

We suppose there are two assets, one bond and one stock, which are traded in the market. Let $d=1, n=$ $1, N=2$, the bond satisfies

$$
\left\{\begin{aligned}
\mathrm{d} R(t) & =r(t) R(t) \mathrm{d} t, \quad t>0, \\
R(0) & =a_{0}>0
\end{aligned}\right.
$$

and the stock asset is described by

$$
\left\{\begin{array}{l}
\mathrm{d} S(t)=b(t) S(t) \mathrm{d} t+\sigma(t) S(t) \mathrm{d} W(t), \quad t>0, \\
S(0)=s_{0}>0 .
\end{array}\right.
$$


Our target is to minimize the following multi-time sate mean-variance problem:

$$
J(\pi(\cdot))=\sum_{i=1}^{2}\left(\frac{\mu_{i}}{2} \operatorname{Var}\left(Y^{\pi}\left(t_{i}\right)\right)-\mathbb{E}\left[Y^{\pi}\left(t_{i}\right)\right]\right),
$$

and a tractable auxiliary problem is given as follows:

$$
\hat{J}(\pi(\cdot))=\sum_{i=1}^{2} \mathbb{E}\left[\frac{\mu_{i}}{2} Y^{\pi}\left(t_{i}\right)^{2}-\lambda_{i} Y^{\pi}\left(t_{i}\right)\right] .
$$

Based on the results in Theorem 3.6 and formulas (3.22) and (3.27), we set

$$
\begin{aligned}
\lambda_{2}^{*}= & e^{\int_{t_{1}}^{t_{2}} \beta(t) \mathrm{d} t}+\mu_{2} \mathbb{E}\left[Y^{*}\left(t_{1}\right)\right] e^{\int_{t_{1}}^{t_{2}} r(t) \mathrm{d} t} ; \\
\lambda_{1}^{*}= & \frac{P_{2}\left(t_{1}\right)+\mu_{1}}{P_{2}\left(t_{1}\right)+\mu_{1} e^{-\int_{0}^{t_{1}} \beta(t) \mathrm{d} t}} \\
& +\mu_{1}\left(\frac{P_{2}\left(t_{1}\right) \rho_{2}-g_{2}\left(t_{1}\right)}{P_{2}\left(t_{1}\right)+\mu_{1} e^{-\int_{0}^{t_{1}} \beta(t) \mathrm{d} t}}\left[1-e^{-\int_{0}^{t_{1}} \beta(t) \mathrm{d} t}\right]\right. \\
& \left.+\frac{P_{2}\left(t_{1}\right)+\mu_{1}}{P_{2}\left(t_{1}\right)+\mu_{1} e^{-\int_{0}^{t_{1}} \beta(t) \mathrm{d} t}} y e^{\int_{0}^{t_{1}}[r(t)-\beta(t)] \mathrm{d} t}\right) .
\end{aligned}
$$

The optimal strategy of model (4.2) is given as follows:

$$
\pi^{*}(t)=\frac{b(t)-r(t)}{\sigma(t)^{2}}\left[\left(\frac{\lambda_{i}^{*}}{\mu_{i}}-\frac{g_{i}\left(t_{i}\right)}{P_{i}\left(t_{i}\right)}\right) e^{-\int_{t}^{t_{i}} r(t) \mathrm{d} t}-Y^{*}(t)\right], t_{i-1}<t \leq t_{i}, i=1,2,
$$

and

$$
\begin{aligned}
& \mathbb{E}\left[Y^{*}\left(t_{2}\right)\right]=\frac{e^{\int_{t_{1}}^{t_{2}} \beta(t) \mathrm{d} t}-1}{\mu_{2}}+\mathbb{E}\left[Y^{*}\left(t_{1}\right)\right] e^{\int_{t_{1}}^{t_{2}} r(t) \mathrm{d} t} ; \\
& \mathbb{E}\left[Y^{*}\left(t_{1}\right)\right]=y e^{\int_{0}^{t_{1}}[r(t)-\beta(t)] \mathrm{d} t}+\left(\frac{\lambda_{1}^{*}}{\mu_{1}}-\frac{P_{2}\left(t_{1}\right)\left(\rho_{1}-\rho_{2}\right)+g_{2}\left(t_{1}\right)}{P_{2}\left(t_{1}\right)+\mu_{1}}\right)\left(1-e^{-\int_{0}^{t_{1}} \beta(t) \mathrm{d} t}\right) ; \\
& \beta(t)=\left(\frac{b(t)-r(t)}{\sigma(t)}\right)^{2}, t \leq t_{2},
\end{aligned}
$$

and $\left(P_{2}\left(t_{1}\right), g_{2}\left(t_{1}\right)\right)$ satisfies the following Riccati equations,

$$
\left\{\begin{array}{l}
\mathrm{d} P_{2}(t)=[\beta(t)-2 r(t)] P_{2}(t) \mathrm{d} t \\
P_{2}\left(t_{2}\right)=\mu_{2}+P_{3}\left(t_{2}\right), t_{1} \leq t<t_{2}
\end{array}\right.
$$

and related equations,

$$
\left\{\begin{array}{l}
\mathrm{d} g_{2}(t)=\left[(\beta(t)-r(t)) g_{2}(t)-\rho_{2} r(t) P_{2}(t)\right] \mathrm{d} t \\
g_{2}\left(t_{2}\right)=g_{3}\left(t_{2}\right)+P_{3}\left(t_{2}\right)\left(\rho_{2}-\rho_{3}\right), t_{1} \leq t<t_{2}
\end{array}\right.
$$


where $P_{3}\left(t_{2}\right)=0, g_{3}\left(t_{2}\right)=0, \rho_{3}=0$. By a simple calculation, we can obtain that

$$
\begin{aligned}
& P_{2}\left(t_{1}\right)=\mu_{2} e^{\int_{t_{1}}^{t_{2}}[2 r(t)-\beta(t)] \mathrm{d} t} ; \\
& \frac{\lambda_{2}^{*}}{\mu_{2}}-\frac{g_{2}\left(t_{2}\right)}{P_{2}\left(t_{2}\right)}=\frac{\lambda_{2}^{*}}{\mu_{2}} ; \\
& \frac{\lambda_{1}^{*}}{\mu_{1}}-\frac{g_{1}\left(t_{1}\right)}{P_{1}\left(t_{1}\right)}=\frac{\lambda_{1}^{*}+\lambda_{2}^{*} e^{\int_{t_{1}}^{t_{2}}[r(t)-\beta(t)] \mathrm{d} t}}{\mu_{1}+\mu_{2} e^{\int_{t_{1}}^{t_{2}}[2 r(t)-\beta(t)] \mathrm{d} t} .}
\end{aligned}
$$

Combining formulas (4.3) and (4.6), it follows that

$$
\begin{aligned}
\mathbb{E}\left[Y^{*}\left(t_{1}\right)\right]= & y e^{\int_{0}^{t_{1}}[r(t)-\beta(t)] \mathrm{d} t}+\frac{\lambda_{1}^{*}+\lambda_{2}^{*} e^{\int_{t_{1}}^{t_{2}}[r(t)-\beta(t)] \mathrm{d} t}}{\mu_{1}+\mu_{2} e^{\int_{t_{1}}^{t_{2}}[2 r(t)-\beta(t)] \mathrm{d} t}}\left[1-e^{-\int_{0}^{t_{1}} \beta(t) \mathrm{d} t}\right] \\
\mathbb{E}\left[Y^{*}\left(t_{2}\right)\right]= & \frac{e^{\int_{t_{1}}^{t_{2}} \beta(t) \mathrm{d} t}-1}{\mu_{2}}+\mathbb{E}\left[Y^{*}\left(t_{1}\right)\right] e^{\int_{t_{1}}^{t_{2}} r(t) \mathrm{d} t} ; \\
\lambda_{2}^{*}= & e^{\int_{t_{1}}^{t_{2}} \beta(t) \mathrm{d} t}+\mu_{2} \mathbb{E}\left[Y^{*}\left(t_{1}\right)\right] e^{\int_{t_{1}}^{t_{2}} r(t) \mathrm{d} t} ; \\
\lambda_{1}^{*}= & \frac{P_{2}\left(t_{1}\right)+\mu_{1}}{P_{2}\left(t_{1}\right)+\mu_{1} e^{-\int_{0}^{t_{1}} \beta(t) \mathrm{d} t}} \\
& +\mu_{1}\left(\frac{P_{2}\left(t_{1}\right) \rho_{2}-g_{2}\left(t_{1}\right)}{P_{2}\left(t_{1}\right)+\mu_{1} e^{-\int_{0}^{t_{1}} \beta(t) \mathrm{d} t}}\left[1-e^{-\int_{0}^{t_{1}} \beta(t) \mathrm{d} t}\right]\right. \\
& +\frac{P_{2}\left(t_{1}\right)+\mu_{1}}{\left.P_{2}\left(t_{1}\right)+\mu_{1} e^{-\int_{0}^{t_{1}} \beta(t) \mathrm{d} t} y e^{\int_{0}^{t_{1}}[r(t)-\beta(t)] \mathrm{d} t}\right)}
\end{aligned}
$$

In the following, we set $T=2, y=1, t_{1}=1$, and $t_{2}=2$. Let $r(t)=r, b(t)=b, \sigma(t)=\sigma, \beta(t)=\beta$, where $0 \leq t \leq T$. From formulas (4.7), we have

$$
\begin{aligned}
\mathbb{E}\left[Y^{*}(1)\right]= & e^{r-\beta}+\frac{\lambda_{1}^{*}+\lambda_{2}^{*} e^{r-\beta}}{\mu_{1}+\mu_{2} e^{2 r-\beta}}\left(1-e^{-\beta}\right) ; \\
\mathbb{E}\left[Y^{*}(2)\right]= & \frac{e^{\beta}-1}{\mu_{2}}+\mathbb{E}\left[Y^{*}\left(t_{1}\right)\right] e^{r} ; \\
\lambda_{2}^{*}= & e^{\beta}+\mu_{2} \mathbb{E}\left[Y^{*}(1)\right] e^{r} ; \\
\lambda_{1}^{*}= & \mu_{1}\left(\frac{\lambda_{2}^{*}\left(e^{r}-e^{r-\beta}\right)}{\mu_{2} e^{2 r}+\mu_{1}}+\frac{\mu_{2} e^{3 r-\beta}+\mu_{1} e^{r}}{\mu_{2} e^{2 r}+\mu_{1}}\right) \\
& +\frac{\mu_{2} e^{2 r}+\mu_{1} e^{\beta}}{\mu_{2} e^{2 r}+\mu_{1}} .
\end{aligned}
$$

Remark 4.1. Let $\mathbb{E}\left[Y^{*}(1)\right]=L_{1}, \mathbb{E}\left[Y^{*}(2)\right]=L_{2}$, and

$$
L_{2}>L_{1} e^{r}>e^{2 r}, \quad\left(L_{2}-L_{1} e^{r}\right) e^{\beta} \neq\left(L_{1}-e^{r}\right) e^{r}
$$

Note that, the condition $L_{2}>L_{1} e^{r}>e^{2 r}$ guarantees that the constraints on the mean values $\mathbb{E}\left[Y^{*}(1)\right]=$ $L_{1}, \mathbb{E}\left[Y^{*}(2)\right]=L_{2}$ are bigger than the return that is invested into the bond, while the condition $\left(L_{2}-L_{1} e^{r}\right) e^{\beta} \neq$ $\left(L_{1}-e^{r}\right) e^{r}$ guarantees the parameter $\mu_{1} \neq 0$. 
Applying formulas (4.8), by a simple calculation, one obtains

$$
\begin{aligned}
\mu_{2}= & \frac{e^{\beta}-1}{L_{2}-L_{1} e^{r}} ; \\
\lambda_{2}^{*}= & \frac{L_{2} e^{\beta}-L_{1} e^{r}}{L_{2}-L_{1} e^{r}} ; \\
\mu_{1}= & \frac{\left(e^{\beta}-1\right)\left(e^{\beta}+\lambda_{2}^{*} e^{r}\right)-\left(L_{1} e^{\beta}-e^{r}\right) e^{2 r} \mu_{2}}{\left(L_{1}-e^{r}\right) e^{\beta}} ; \\
\lambda_{1}^{*}= & \mu_{1}\left(\frac{\lambda_{2}^{*}\left(e^{r}-e^{r-\beta}\right)}{\mu_{2} e^{2 r}+\mu_{1}}+\frac{\mu_{2} e^{3 r-\beta}+\mu_{1} e^{r}}{\mu_{2} e^{2 r}+\mu_{1}}\right) \\
& +\frac{\mu_{2} e^{2 r}+\mu_{1} e^{\beta}}{\mu_{2} e^{2 r}+\mu_{1}} .
\end{aligned}
$$

Based on Theorem 3.6, applying the formula (4.6), we can obtain the related optimal strategy for the multi-time state mean-variance model (4.1) with the constraints on means $\mathbb{E}\left[Y^{*}(1)\right]=L_{1}, \mathbb{E}\left[Y^{*}(2)\right]=L_{2}$,

$$
\pi^{*}(t)=\left\{\begin{array}{l}
\frac{b-r}{\sigma^{2}}\left[\frac{\lambda_{1}^{*}+\lambda_{2}^{*} e^{r-\beta}}{\mu_{1}+\mu_{2} e^{2 r-\beta}} e^{r(t-1)}-Y^{*}(t)\right], 0 \leq t \leq 1 ; \\
\frac{b-r}{\sigma^{2}}\left[\frac{\lambda_{2}^{*}}{\mu_{2}} e^{r(t-2)}-Y^{*}(t)\right], 1<t \leq 2 .
\end{array}\right.
$$

Thus, $\mathbb{E}\left[Y^{*}(\cdot)\right]$ satisfies

$$
\mathbb{E}\left[Y^{*}(t)\right]=\left\{\begin{array}{l}
e^{(r-\beta) t}+\frac{\lambda_{1}^{*}+\lambda_{2}^{*} e^{r-\beta}}{\mu_{1}+\mu_{2} e^{2 r-\beta}}\left[e^{r(t-1)}-e^{(r-\beta) t-r}\right], 0 \leq t \leq 1 ; \\
\mathbb{E}\left[Y^{*}(1)\right] e^{(r-\beta)(t-1)}+\frac{\lambda_{2}^{*}}{\mu_{2}}\left[e^{r(t-2)}-e^{r(t-2)-\beta(t-1)}\right], 1<t \leq 2,
\end{array}\right.
$$

and from Lemma 3.4, the variances of $Y^{*}(\cdot)$ at $t_{1}=1, t_{2}=2$ are given as follows:

$$
\begin{aligned}
& \operatorname{Var}\left(Y^{*}(1)\right)=\frac{\left(\mathbb{E}\left[Y^{*}(1)\right]-e^{r}\right)^{2}}{e^{\beta}-1} ; \\
& \operatorname{Var}\left(Y^{*}(2)\right)=\operatorname{Var}\left(Y^{*}(1)\right) e^{2 r-\beta}+\frac{\left(\mathbb{E}\left[Y^{*}(2)\right]-\mathbb{E}\left[Y^{*}(1)\right] e^{r}\right)^{2}}{e^{\beta}-1} .
\end{aligned}
$$

Now, we show the results of case $N=1$, which is the classical mean-variance model:

$$
\mathbb{E}\left[Y^{\#}(2)\right]=L_{2}, \quad \mu=\frac{e^{2 \beta}-1}{L_{2}-e^{2 r}}, \lambda^{*}=\frac{L_{2} e^{2 \beta}-e^{2 r}}{L_{2}-e^{2 r}},
$$

where the related optimal strategy is

$$
\pi^{\#}(t)=\frac{b-r}{\sigma^{2}}\left[\frac{\lambda^{*}}{\mu} e^{r(t-2)}-Y^{\#}(t)\right], 0 \leq t \leq 2 .
$$


The mean $\mathbb{E}\left[Y^{\#}(\cdot)\right]$ and variance $\operatorname{Var}\left(Y^{\#}(2)\right)$ satisfy

$$
\begin{aligned}
& \mathbb{E}\left[Y^{\#}(t)\right]=e^{(r-\beta) t}+\frac{\lambda^{*} e^{r(t-2)}}{\mu}\left[1-e^{-\beta t}\right] ; \\
& \operatorname{Var}\left(Y^{\#}(2)\right)=\frac{\left(\mathbb{E}\left[Y^{\#}(2)\right]-e^{2 r}\right)^{2}}{e^{2 \beta}-1},
\end{aligned}
$$

where $\mathbb{E}\left[Y^{\#}(2)\right]=L_{2}$. Based on formulas (4.12) and (4.14), we have the following comparison results for $\left(\mathbb{E}\left[Y^{*}(1)\right], \operatorname{Var}\left(Y^{*}(2)\right)\right)$ and $\left(\mathbb{E}\left[Y^{\#}(1)\right], \operatorname{Var}\left(Y^{\#}(2)\right)\right)$ :

Corollary 4.2. Suppose $L_{1}$ and $L_{2}$ satisfy condition (4.9), one obtains

$$
\operatorname{Var}\left(Y^{*}(2)\right)>\operatorname{Var}\left(Y^{\#}(2)\right) \text {. }
$$

Moreover, when $\left(L_{2}-L_{1} e^{r}\right) e^{\beta}>\left(L_{1}-e^{r}\right) e^{r}$, we have

$$
\mathbb{E}\left[Y^{*}(1)\right]<\mathbb{E}\left[Y^{\#}(1)\right]
$$

when $\left(L_{2}-L_{1} e^{r}\right) e^{\beta}<\left(L_{1}-e^{r}\right) e^{r}$, we have

$$
\mathbb{E}\left[Y^{*}(1)\right]>\mathbb{E}\left[Y^{\#}(1)\right]
$$

Proof. By formula (4.12), we have

$$
\begin{aligned}
\operatorname{Var}\left(Y^{*}(2)\right) & =\frac{\left(L_{1}-e^{r}\right)^{2}}{e^{\beta}-1} e^{2 r-\beta}+\frac{\left(L_{2}-L_{1} e^{r}\right)^{2}}{e^{\beta}-1} \\
& =\frac{\left[e^{2 r-\beta}+e^{2 r}\right] L_{1}^{2}-2 e^{r}\left[L_{2}+e^{2 r-\beta}\right] L_{1}+L_{2}^{2}+e^{4 r-\beta}}{e^{\beta}-1} \\
& =\frac{e^{2 r-\beta}+e^{2 r}}{e^{\beta}-1}\left[L_{1}-\frac{L_{2} e^{\beta-r}+e^{r}}{e^{\beta}+1}\right]^{2}+\frac{\left(L_{2}-e^{2 r}\right)^{2}}{e^{2 \beta}-1}
\end{aligned}
$$

From equality (4.14), one obtains

$$
\operatorname{Var}\left(Y^{\#}(2)\right)=\frac{\left(L_{2}-e^{2 r}\right)^{2}}{e^{2 \beta}-1} .
$$

It follows that

$$
\operatorname{Var}\left(Y^{*}(2)\right)>\operatorname{Var}\left(Y^{\#}(2)\right)
$$

By equality (4.14), we have

$$
\mathbb{E}\left[Y^{\#}(1)\right]=\frac{L_{2} e^{\beta-r}+e^{r}}{e^{\beta}+1} .
$$

Based on the equality $\left(L_{2}-L_{1} e^{r}\right) e^{\beta}>\left(L_{1}-e^{r}\right) e^{r}$ and Remark 4.1, one obtains

$$
e^{r}<\mathbb{E}\left[Y^{*}(1)\right]=L_{1}<\frac{L_{2} e^{\beta-r}+e^{r}}{e^{\beta}+1}=\mathbb{E}\left[Y^{\#}(1)\right],
$$


and $\mathbb{E}\left[Y^{*}(1)\right]>\mathbb{E}\left[Y^{\#}(1)\right]$ when $\left(L_{2}-L_{1} e^{r}\right) e^{\beta}<\left(L_{1}-e^{r}\right) e^{r}$. This completes the proof.

Example 4.3. Let $b, r, \sigma$ be constants, and $T=2, y=1, b=2 r, \beta=r, L_{1}=2 e^{r}, L_{2}=3 e^{2 r}$. Now, we consider two special cases of the model in this part: $N=1,2$. For the case $N=2, t_{1}=1$, and $t_{2}=2$, by (4.10), (4.11), and (4.12). We have

$$
\mu_{1}=e^{r}-1 ; \lambda_{1}^{*}=2 e^{2 r}-2 e^{r}+1 ; \mu_{2}=e^{-r}-e^{-2 r} ; \lambda_{2}^{*}=3 e^{r}-2,
$$

and

$$
\pi^{*}(t)=\left\{\begin{array}{l}
\frac{2 e^{r}-1}{e^{r}-1} e^{r t}-Y^{*}(t), 0 \leq t \leq 1 \\
\frac{3 e^{r}-2}{e^{r}-1} e^{r t}-Y^{*}(t), 1<t \leq 2
\end{array}\right.
$$

The explicit formula of $\mathbb{E}\left[Y^{*}(\cdot)\right]$ is

$$
\mathbb{E}\left[Y^{*}(t)\right]=\left\{\begin{array}{l}
1+\frac{2 e^{r}-1}{e^{r}-1}\left[e^{r t}-1\right], 0 \leq t \leq 1 ; \\
\mathbb{E}\left[Y^{*}(1)\right]+\frac{3 e^{r}-2}{e^{r}-1}\left[e^{r t}-e^{r}\right], 1<t \leq 2,
\end{array}\right.
$$

and the variances of $Y^{*}(\cdot)$ at $t_{1}=1, t_{2}=2$ are given as follows:

$$
\operatorname{Var}\left(Y^{*}(1)\right)=\frac{e^{2 r}}{e^{r}-1} ; \operatorname{Var}\left(Y^{*}(2)\right)=\frac{e^{3 r}+e^{4 r}}{e^{r}-1} .
$$

For the case $N=1$, which is the classical mean-variance model, the explicit formulas of the optimal asset and related parameters are

$$
\mathbb{E}\left[Y^{\#}(2)\right]=3 e^{2 r}, \mu=\frac{e^{2 r}-1}{2 e^{2 r}}, \lambda^{*}=\frac{3 e^{2 r}-1}{2},
$$

and the related optimal strategy is

$$
\pi^{\#}(t)=\frac{3 e^{2 r}-1}{e^{2 r}-1} e^{r t}-Y^{\#}(t), 0 \leq t \leq 2 .
$$

The mean $\mathbb{E}\left[Y^{\#}(\cdot)\right]$ and variance $\operatorname{Var}\left(Y^{\#}(\cdot)\right)$ satisfy

$$
\mathbb{E}\left[Y^{\#}(t)\right]=1+\frac{3 e^{2 r}-1}{e^{2 r}-1}\left[e^{r t}-1\right] ; \operatorname{Var}\left(Y^{\#}(2)\right)=\frac{4 e^{4 r}}{e^{2 r}-1} .
$$

Based on formulas (4.17) and (4.19), we have the comparison results for $\left(\mathbb{E}\left[Y^{*}(1)\right], \operatorname{Var}\left(Y^{*}(2)\right)\right)$ and $\left(\mathbb{E}\left[Y^{\#}(1)\right], \operatorname{Var}\left(Y^{\#}(2)\right)\right)$ :

$$
\mathbb{E}\left[Y^{*}(1)\right]<\mathbb{E}\left[Y^{\#}(1)\right] ; \quad \operatorname{Var}\left(Y^{*}(2)\right)>\operatorname{Var}\left(Y^{\#}(2)\right),
$$

which verifies the condition $\left(L_{2}-L_{1} e^{r}\right) e^{\beta}>\left(L_{1}-e^{r}\right) e^{r}$ and the results of Corollary 4.2. Furthermore, we can take other values of $L_{1}$ and $L_{2}$ such that $\left(L_{2}-L_{1} e^{r}\right) e^{\beta}<\left(L_{1}-e^{r}\right) e^{r}$, which is used to verify the results of Corollary 4.2 . 


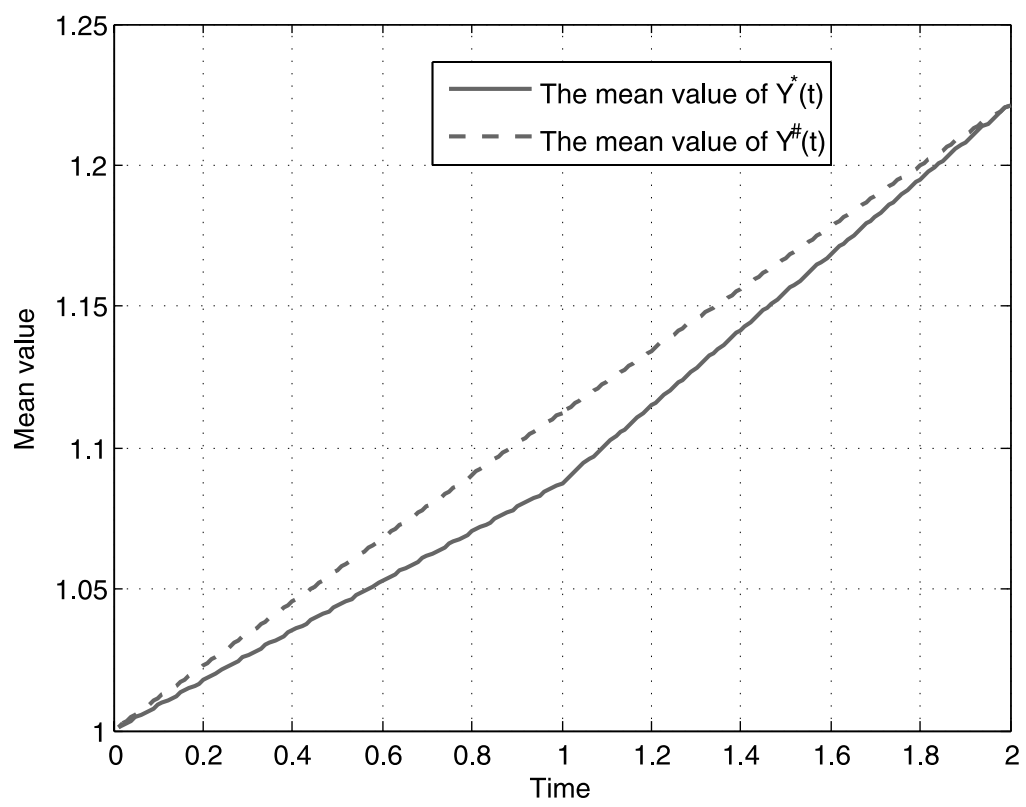

Figure 1. Comparing the values of $\mathbb{E}\left[Y^{*}(\cdot)\right]$ and $\mathbb{E}\left[Y^{\#}(\cdot)\right]$. $\mathbb{E}\left[Y^{*}(\cdot)\right]$ is the expectation of the optimal state $Y^{*}(\cdot)$ under two-time state mean-variance model, and $\mathbb{E}\left[Y^{\#}(\cdot)\right]$ is the expectation of the optimal state $Y^{\#}(\cdot)$ under one-time state mean-variance model.

As we have shown in this example, we can use the multi-time state mean-variance model to control the average return of the asset before terminal time $T$. In particular, when $N=2$, we want to minimize the variances of the asset at time $t_{1}$ and $t_{2}$ with the given returns $L_{1}, L_{2}$ at time $t_{1}$ and $t_{2}$. Thus, the advantages of the multi-time state mean-variance model are that we can adjust the return at $t_{1}$ and minimize the variance at time $t_{1}$.

\subsection{Simulation analysis}

Let $r=0.04, b=0.12, \sigma=0.2, \beta=0.16$, we show the simulation results of the case $N=2$, and case $N=1$, where case $N=1$ is same with the classical continuous time mean-variance model.

In Figure 1, we take $L_{2}=e^{5 r}, L_{1}=e^{2.1 r}$, which satisfies conditions (4.9). The expectations of $Y^{*}(\cdot)$ and $Y^{\#}(\cdot)$ are given as follows, respectively,

$$
\mathbb{E}\left[Y^{*}(t)\right]=\left\{\begin{array}{l}
e^{(r-\beta) t}+\frac{\lambda_{1}^{*}+\lambda_{2}^{*} e^{r-\beta}}{\mu_{1}+\mu_{2} e^{2 r-\beta}}\left[e^{r(t-1)}-e^{(r-\beta) t-r}\right], 0 \leq t \leq 1 ; \\
\mathbb{E}\left[Y^{*}(1)\right] e^{(r-\beta)(t-1)}+\frac{\lambda_{2}^{*}}{\mu_{2}}\left[e^{r(t-2)}-e^{r(t-2)-\beta(t-1)}\right], 1<t \leq 2,
\end{array}\right.
$$

and

$$
\mathbb{E}\left[Y^{\#}(t)\right]=e^{(r-\beta) t}+\frac{\lambda^{*} e^{r(t-2)}}{\mu}\left[1-e^{-\beta t}\right], 0 \leq t \leq 2 .
$$

From conditions (4.9), we obtain $\mathbb{E}\left[Y^{*}(1)\right]=L_{1}<\mathbb{E}\left[Y^{\#}(1)\right]$ and thus,

$$
\mathbb{E}\left[Y^{*}(t)\right]<\mathbb{E}\left[Y^{\#}(t)\right], 0<t<2 .
$$



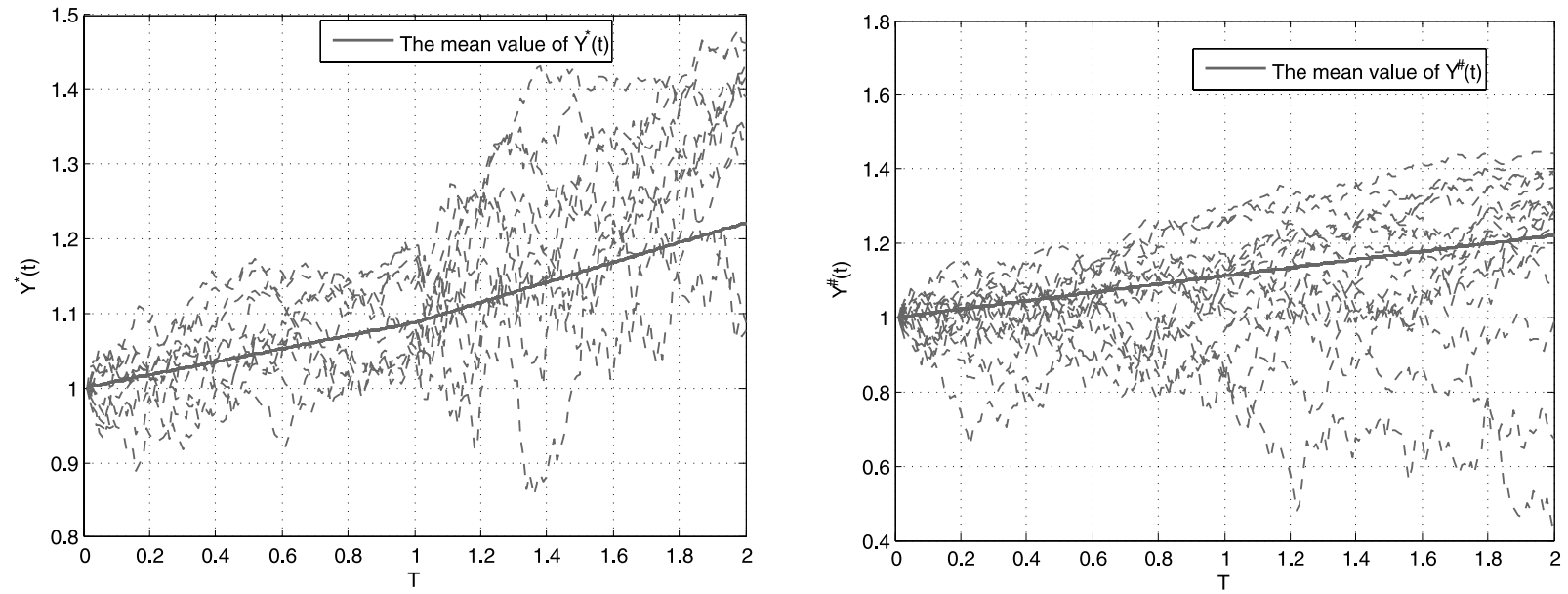

Figure 2. Comparing the values of $Y^{*}(\cdot)$ and $Y^{\#}(\cdot)$. The left map plots the pathwise value of $Y^{*}(\cdot)$ along with the mean value of $Y^{*}(\cdot)$, and the right map plots the pathwise value of $Y^{\#}(\cdot)$ along with the mean value of $Y^{\#}(\cdot)$.

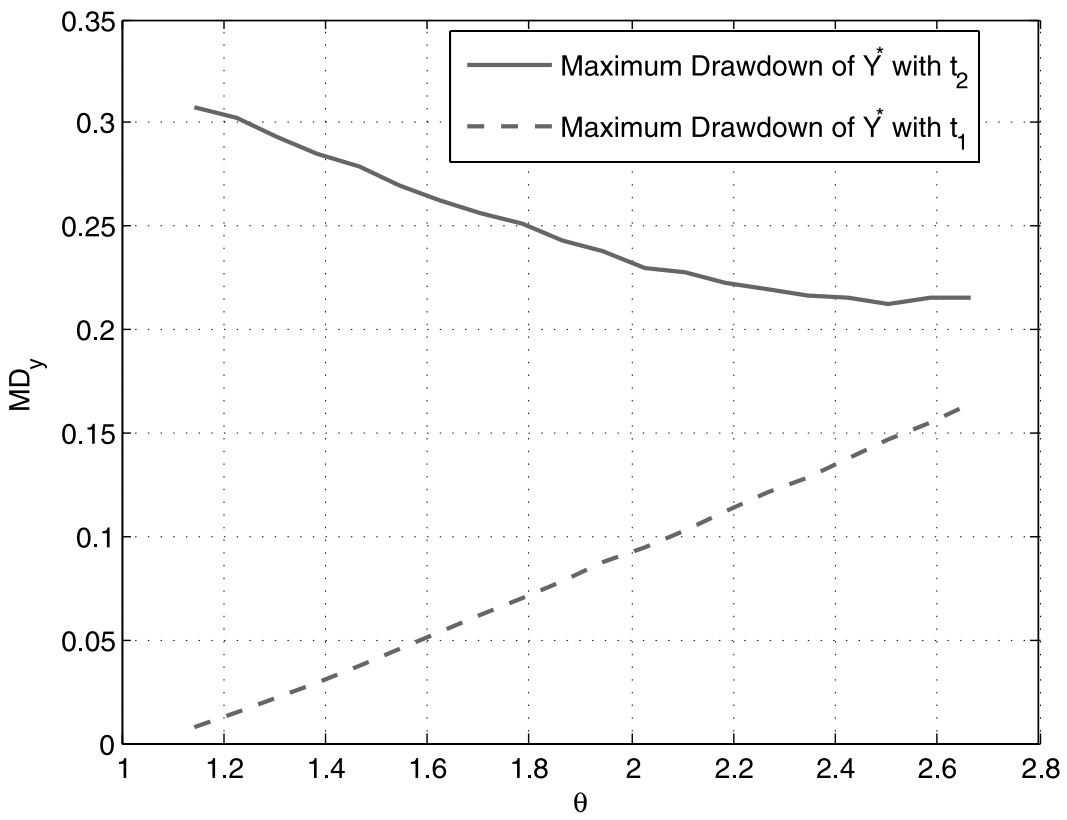

Figure 3. The average Maximum-Drawdown of $Y^{*}(\cdot)$ along with $\theta$. We plot the Maximum Drawdown of $Y^{*}(\cdot)$ with the parameter $\theta$ under different terminal times $t_{2}=2$ and $t_{1}=1$.

These results show that if we want to minimize the variances of the wealth at times $t_{1}=1, t_{2}=2$ together, the means of the investment portfolio may be smaller than that of the classical mean-variance model in continuous time.

In Figure 2, we plot the values $Y^{*}(\cdot)$ and $Y^{\#}(\cdot)$ in pathwise. The left shows the pathwise of the function $Y^{*}(\cdot)$ along with $\mathbb{E}\left[Y^{*}(\cdot)\right]$, while the right shows that of $Y^{\#}(\cdot)$. We can see that the variance of $Y^{*}(1)$ is bigger than that of $Y^{*}(1)$, and the variance of $Y^{*}(2)$ is almost the same as that of $Y^{*}(2)$. These phenomena verify the 
results of Corollary 4.2. In addition, in Figure 1, we can see that $\mathbb{E}\left[Y^{*}(t)\right]<\mathbb{E}\left[Y^{\#}(t)\right], 0<t \leq 1$, while Figure 2 shows that the variance of $Y^{*}(\cdot)$ is smaller than that of $Y^{\#}(\cdot)$ before time 1.

In Figure 3, we plot the function of $\mathbb{E}\left[\mathrm{MD}_{Y^{*}}^{h}\right]$ along with $\theta \in[1.145,2.665]$, where

$$
\operatorname{MD}_{Y^{*}}^{h}=\operatorname{esssup}\left\{z \mid z=Y^{*}(t)-Y^{*}(s), 0 \leq t \leq s \leq h\right\},
$$

$0<h \leq 2$, and

$$
L_{1}=e^{\theta r}, \quad \mathbb{E}\left[Y^{\#}(1)\right]=e^{2.665 r} .
$$

We can see that $\mathbb{E}\left[\mathrm{MD}_{Y^{*}}^{t_{2}}\right]$ is decreasing with $\theta \in[1.145,2.505]$, increasing with $\theta \in[2.505,2.665]$ and thus decreasing with $L_{1} \in\left[e^{1.145 r}, e^{2.505 r}\right]$ and increasing with $L_{1} \in\left[e^{2.505 r}, e^{2.665 r}\right]$, while $\mathbb{E}\left[\mathrm{MD}_{Y^{*}}^{t_{1}}\right]$ is increasing with $L_{1} \in\left[e^{1.145 r}, e^{2.665 r}\right]$, where $t_{1}=1, t_{2}=2$.

\section{CONCLUSiON}

For given $0=t_{0}<t_{1}<\cdots<t_{N}=T$, to reduce the variance of the mean-variance model at the multitime state $\left(Y^{\pi}\left(t_{1}\right), \ldots, Y^{\pi}\left(t_{N}\right)\right)$, we propose a multi-time state mean-variance model with a constraint on the multi-time state mean value. In the proposed model, we solve the multi-time state mean-variance model by introducing a sequence of Riccati equations.

The main results of this study are as follows:

- We can use the multi-time state mean-variance model to manage the risk of the investment portfolio along the multi-time $0=t_{0}<t_{1}<\cdots<t_{N}=T$.

- A sequence of Riccati equations that are connected by jump boundary conditions are introduced, on which we obtain an optimal strategy for the multi-time state mean-variance model.

- Furthermore, the relationships of the means and variances of this multi-time state mean-variance model are established, similar to the one in the classical mean-variance model.

- A simulation is employed to show that minimizing the variances for multi-time state can affect the average value of Maximum-Drawdown of the investment portfolio.

Acknowledgements. The author are grateful to numerous detailed suggestions and comments from two reviewers and the editor that have led to significant improvements of the paper.

\section{REFERENCES}

[1] I. Bajeux-Besnainou and R. Portait, Dynamic asset allocation in a mean-variance framework. Manag. Sci. 11 (1998) 79-95.

[2] S. Basak and G. Chabakauri, Dynamic mean-variance asset allocation. Rev. Financial Stud. 23 (2010) $2970-3016$.

[3] A. Bensoussan, K. Sung and S.C.P. Yam, Linear-quadratic time-inconsistent mean field games. Dyn. Games. Appl. 3 (2013) $537-552$.

[4] A. Bensoussan, K. Sung, S.C.P. Yam and S.P. Yung, Linear-quadratic mean field games. J. Optim. Theory Appl. 169 (2016) 496-529.

[5] T.R. Bielecki, H.Q. Jin, S. Pliska and X.Y. Zhou, Continuous-time mean-variance portfolio selection with bankruptcy prohibition. Math. Finance 15 (2005) 213-244.

[6] T. Björk, A. Murgoci and X.Y. Zhou, Mean-variance protfolio optimization with state-dependent risk aversion. Math. Finance 24 (2014) 1-24.

[7] T. Björk, M. Khapko and A. Murgoci, On time-inconsistent stochastic control in continuous time. Finance Stoch. 21 (2017) 331-360.

[8] C. Christoph, Time-consistent mean-variance portfolio selection in discrete and continuous time. Finance Stoch. 17 (2013) $227-271$.

[9] M. Dai, Z.Q. Xu and X.Y. Zhou, Continuous-time Markowitz's model with transaction costs. SIAM J. Financial Math. 1 (2010) 96-125.

[10] M. Dai, H. Jin, K. Steven and Y. Xu, A dynamic mean-variance analysis for log returns. Manag. Sci. 67 (2020) 1-16.

[11] Y. Hu, H.Q. Jin and X.Y. Zhou, Time-inconsistent stochastic linear-quadratic control. SIAM J. Control Optim. 50 (2012) $1548-1572$ 
[12] M. Huang, P.E. Caines and R.P. Malhame, The Nash certainty equivalence principle and McKean-Vlasov systems: an invariance principle and entry adaptation, in Proceedings of the 46th IEEE Conference on Decision and Control (2007) 121-126.

[13] C. Karnam, J. Ma and J. Zhang, Dynamic approaches for some time-inconsistent optimization problems. Ann. Appl. Probab. 27 (2017) 3435-3477.

[14] G. Kovácová and B. Rudloff, Time consistency of the mean-risk problem. Oper. Res. (2020) 1-37.

[15] F.E. Kydland and E. Prescott, Rules rather than discretion: the inconsistency of optimal plans. J. Polit. Econ. 85 (1997) 473-492.

[16] D. Li and W.L. Ng, Optimal dynamic portfolio selection: Multi-period mean-variance formulation. Math. Finance 10 (2000) $387-406$.

[17] A.E.B. Lim, Quadratic hedging and mean-variance portfolio selection with random parameters in an incomplete market. Math. Oper. Res. 29 (2004) 132-161.

[18] A.E.B. Lim and X.Y. Zhou, Quadratic hedging and mean-variance portfolio selection with random parameters in a complete market. Math. Oper. Res. 1 (2002) 101-120.

[19] H. Markowitz, Portfolio selection. J. Finance 7 (1952) 77-91.

[20] H. Markowitz, Portfolio Selection: Efficient Diversification of Investment. John Wiley \& Sons, New York (1959).

[21] L. Martellini and B. Urošević, Static mean-variance analysis with uncertain time horizon. Manag. Sci. 52 (2006) 955-964.

[22] R.C. Merton, An analytic derivation of the efficient frontier. J. Finance Quant. Anal. 7 (1972) 1851-1872.

[23] C. Pun, Robust time-inconsistent stochastic control problems. Automatica 94 (2018) 249-257.

[24] H.R. Richardson, A minimum variance result in continuous trading portfolio optimization. Manag. Sci. 9 (1989) 1045-1055.

[25] Y. Shen, Mean-variance portfolio selection in a complete market with unbounded random coefficients. Automatica 55 (2015) $165-175$.

[26] H.L. Wu, Z.F. Li and D. Li, Multi-period mean-variance portfolio selection with Markov regime switching and uncertain time horizon. J. Syst. Sci. Complex 24 (2011) 140-155.

[27] J.M. Xia, Mean-variance portfolio choice: Quadratic partial hedging. Math. Finance 15 (2005) 533-538.

[28] T. Yan and H. Wong, Open-loop equilibrium strategy for mean-variance portfolio problem under stochastic volatility. Automatica 107 (2019) 211-223.

[29] S.Z. Yang, The necessary and sufficient conditions for stochastic differential systems with multi-time state cost functional. Syst. Control Lett. 114 (2018) 11-18.

[30] H. Yao and Q. Ma, Continuous time mean-variance model with uncertain exit time. International Conference on Management and Service Science, Wuhan (2010) 1-4.

[31] L. Yi, Z.F. Li and D. Li, Multi-period portfolio selection for asset-liability management with uncertain investment horizon. $J$. Ind. Manag. Optim. 4 (2008) 535-552.

[32] Z.Y. Yu, Continuous time mean-variance portfolio selection with random horizon. Appl. Math. Optim. 68 (2013) 333-359.

[33] X.Y. Zhou and D. Li, Continuous-time mean-variance portfolio selection: A stochastic LQ framework. Appl. Math. Optim. 42 (2000) 19-33.

\section{Subscribe to Open (S2O) A fair and sustainable open access model}

This journal is currently published in open access under a Subscribe-to-Open model (S2O). S2O is a transformative model that aims to move subscription journals to open access. Open access is the free, immediate, online availability of research articles combined with the rights to use these articles fully in the digital environment. We are thankful to our subscribers and sponsors for making it possible to publish this journal in open access, free of charge for authors.

\section{Please help to maintain this journal in open access!}

Check that your library subscribes to the journal, or make a personal donation to the $\mathrm{S} 2 \mathrm{O}$ programme, by contacting subscribers@edpsciences.org

More information, including a list of sponsors and a financial transparency report, available at: https://www.edpsciences.org/en/maths-s2o-programme 